\title{
Radio spectral study of the cluster of galaxies Abell 2255
}

\author{
R. F. Pizzo ${ }^{1}$ and A. G. de Bruyn ${ }^{1,2}$ \\ 1 Kapteyn Institute, Postbus 800, 9700 AV Groningen, The Netherlands \\ e-mail: pizzo@astro.rug.nl \\ 2 ASTRON, Postbus 2, 7990 AA Dwingeloo, The Netherlands
}

Received 11 May 2009 / Accepted 31 August 2009

\section{ABSTRACT}

\begin{abstract}
Context. The study of the nonthermal components associated with the intra cluster medium (ICM) in galaxy clusters is important in understanding the history and evolution of clusters.

Aims. Spectral index studies of halos, relics, and radio galaxies provide useful information on their origin and connection with merger processes. Moreover, they reveal the energy spectrum of the relativistic particles and the magnetic field distribution in galaxy clusters. Methods. We present WSRT multi-wavelength observations of the galaxy cluster Abell 2255 at $25 \mathrm{~cm}, 85 \mathrm{~cm}$, and $2 \mathrm{~m}$. The spectral index images allowed us to study the integrated spectrum of halo and relic and to investigate the physical properties of the Beaver head-tail radio galaxy belonging to the cluster.

Results. In the radio halo, the spectral index is steeper at the center and flatter at the locations of the radio filaments, clearly detected at $25 \mathrm{~cm}$. In the relics, the spectral index flattens, moving away from the cluster center. For the Beaver radio galaxy, the spectrum severely steepens from the head towards the end of the tail, because of the energy losses suffered by the relativistic particles. In the $2 \mathrm{~m}$ map, which is the first high-sensitivity image presented in the literature at such a long wavelength, a new Mpc-size emission region is detected between the known radio halo and the NW relic. Not detecting this feature in the more sensitive $85 \mathrm{~cm}$ observations implies that it must have a very steep spectrum $(\alpha \leq-2.6)$.

Conclusions. The observational properties of the radio halo suggest that either we are looking at a superposition of different structures (filaments in the foreground plus real halo in the background) seen in projection across the cluster center or that the halo is intrinsically peculiar. The newly detected extended region to the NW of the halo could be considered as an asymmetric extension of the halo itself. However, since radio halos are known in the literature as structures showing a regular morphology, the new feature could represent the first example of steep Mpc-size diffuse structures (MDS), detected around clusters at very low frequencies. The spectrum of the initial part of the tail of the Beaver, detected at the three wavelengths, is well-fitted by a single injection model.
\end{abstract}

Key words. galaxies: clusters: general - galaxies: clusters: individual: Abell 2255 - galaxies: intergalactic medium

\section{Introduction}

Galaxy clusters, which contains up to a few thousand galaxies and considerable amounts of gas, are the most massive gravitationally bound objects in the universe. Cosmological simulations (Navarro et al. 1995) show that they are not static, but instead grow and still form at the present epoch, as the result of several merger events (e.g. Evrard \& Gioia 2002).

In an increasing number of massive, merging, and X-ray luminous galaxy clusters, large diffuse radio sources associated with the ICM have been detected. They represent the most spectacular aspect of cluster radio emission, and they cannot be associated with any individual galaxy. These diffuse synchrotron radio sources are characterized by a typical size of about $1 \mathrm{Mpc}$, low surface brightness $\left(\sim 1 \mu \mathrm{Jy} \operatorname{arcsec}^{-2}\right.$ at $\left.20 \mathrm{~cm}\right)$, and steep radio spectrum $\left(\alpha \leq-1, S(v) \propto v^{\alpha}\right)$. They are classified (Feretti \& Giovannini 1996) as radio halos, if located at the center of the cluster and not significantly polarized, and radio relics, if lying at the cluster periphery and showing high polarization percentages $(\sim 10 \%-30 \%)$. The synchrotron nature of their radio emission indicates cluster-wide magnetic fields of approximately $0.1-1 \mu \mathrm{G}$, and of a population of relativistic electrons with Lorentz factor $\gamma \gg 1000$.

The knowledge of the physical conditions in halos and relics is important for a comprehensive physical description of the ICM. Since they are related to other cluster properties in the optical and X-ray domains, these extended features are directly connected to the cluster history and evolution. Moreover, they provide a significant test for several theories concerning the origin of relativistic particles in the ICM and particle propagation in astrophysical plasmas.

The origin of halos and relics is still matter of debate. It is suggested that relics relics are related to shocks either by Fermi-I diffuse acceleration of ICM electrons (Enßlin et al. 1998; Keshet et al. 2004b) or by adiabatic energization of the relativistic electrons confined in fossil radio plasma ("ghosts"), released by a former active radio galaxy (Enßlin \& Gopal-Krishna 2001). In the case of radio halos, it is required that the electrons are either re-accelerated (primary models: e.g. Tribble 1993; Brunetti et al. 2001; Petrosian 2001; Brunetti et al. 2004; Fujita et al. 2003; Cassano \& Brunetti 2005) or continuously injected over the entire cluster volume by hadronic collisions (secondary models: e.g. Dennison 1980; Blasi \& Colafrancesco 1999; Dolag \& Enßlin 2000).

Spectral index studies of halos and relics provide important information on their formation and their connection to cluster merger processes. Given their large angular size and steep spectra, low-frequency radio observations are suitable for such investigations. There are only a few clusters for which spectral index maps of halos have been published: Coma (Giovannini et al. 1993), A2163, A665 (Feretti et al. 2004), 
A3562 (Giacintucci et al. 2005), A2744, and A2219 (Orrú et al. 2007). In agreement with primary models, in all these objects the spectral index maps show a patchy structure with, in some cases, a steepening of the spectrum with increasing distance from the center to the edge. The detailed spectral index distribution is known for the relics of A3667 (Rottgering et al. 1997), the Coma cluster (Giovannini et al. 1991), S753 (Subrahmanyan et al. 2003), A2744 and A2219 (Orrú et al. 2007), and A2345 and A1240 (Bonafede et al. 2009). Apart from one of the two relics of A2345, in all the other examples the spectral index flattens going from the regions closer to the cluster center to the outer rim. This is consistent with the presence of electron re-acceleration in an expanding merger shock.

Other important members of clusters in the radio domain are the radio galaxies. Their emission often extends well beyond the optical boundaries of the radio galaxies, out to hundreds of kilo-parsec, and hence it is expected that the ICM would affect their structure. This interaction is proved by the existence of radio galaxies showing distorted structures (tailed radio sources). Spectral studies of radio galaxies with different morphologies at different frequencies provide an excellent opportunity to test the ageing of the electrons, under some standard assumptions.

A2255 is a nearby $(z=0.0806$, Struble \& Rood 1999) and rich cluster, which has been studied in several bands. ROSAT X-ray observations indicate that A2255 has recently undergone a merger (Burns et al. 1995; Feretti et al. 1997; Davis et al. 2003). Recent XMM-Newton observations revealed temperature asymmetries of the ICM suggesting that the merger happened $\sim 0.15$ Gyr ago, probably along the $E-W$ direction (Sakelliou \& Ponman 2006). Optical studies of A2255 revealed the presence of kinematic substructures in the form of several associated groups (Yuan et al. 2003). This result, together with the large ratio of velocity dispersion to X-ray temperature $(6.3 \mathrm{keV}$; Horner 2001) indicates a non-relaxed system.

When studied at radio wavelengths, A2255 shows the presence of a diffuse radio halo (located at the center of the cluster) and of a relic (at the cluster periphery), together with a large number of embedded head-tail radio galaxies (Harris et al. 1980). High resolution radio observations at $21 \mathrm{~cm}$ shown that the radio halo has a rectangular shape and a surface brightness increasing from the center to the edge (Feretti et al. 1997). At this location 3 highly polarized $(\sim 20 \%-40 \%)$ bright filaments perpendicular to each other are detected (Govoni et al. 2005). Sensitive low-frequency observations carried out with the Westerbork synthesis radio telescope (WSRT) at $85 \mathrm{~cm}$ proved the presence of a large number of low-surface brightness extended features around the cluster, that are likely related to Large Scale Structure (LSS) shocks (Pizzo et al. 2008). In this work we combine these data with observations at $25 \mathrm{~cm}$ and at $2 \mathrm{~m}$ in order to study the spectral index properties of the structures belonging to A2255.

This paper is organized as follows. Section 1 describes the main steps of the data reduction and discusses a few issues related to low-frequency radio observations. In Sect. 3, we present the final maps of A2255 at the three wavelengths and in Sect. 4 we show the spectral index analysis for the halo and the relics. In Sect. 5, we analyze the physical properties of the Beaver radio galaxy and we test the ageing processes of the radiating electrons along the tail. We discuss the implications of our results in Sect. 6 and summarize our work in Sect. 7.

Throughout this paper we assume a $\Lambda$ cosmology with $H_{0}=$ $71 \mathrm{~km} \mathrm{~s}^{-1} \mathrm{Mpc}^{-1}, \Omega_{\mathrm{m}}=0.3$, and $\Omega_{\Lambda}=0.7$. All positions are given in $\mathrm{J} 2000$ coordinates. The resolutions are expressed in $\mathrm{RA} \times$ Dec. At the distance of A2255, 1' corresponds to $90 \mathrm{kpc}$.
Table 1. The flux calibrators used during the data reduction.

\begin{tabular}{cccc}
\hline \hline Wavelength & Name & $\begin{array}{c}\text { Flux } \\
\text { (Jy) }\end{array}$ & $\alpha$ \\
\hline $25 \mathrm{~cm}$ & CTD93 & 5.2 & -0.5 \\
$85 \mathrm{~cm}$ & 3C 295 & 63 & -0.6 \\
$2 \mathrm{~m}$ & 3C 295 & 95 & -0.6 \\
\hline
\end{tabular}

\section{Observations and data reduction}

The observations were carried out with the WSRT in three broad frequency ranges with central wavelengths of about $25 \mathrm{~cm}$, $85 \mathrm{~cm}$, and $2 \mathrm{~m}$. The array consists of fourteen $25 \mathrm{~m}$ dishes on an east-west baseline and uses earth rotation to fully synthesize the uv-plane. Ten of the telescopes are on fixed mountings, $144 \mathrm{~m}$ apart; the four remaining dishes are movable along two rail tracks. The interferometer can observe in different configurations that differ from each other in the distance between the last fixed telescope and the first movable one (RT9-RTA). In the array, the baselines can extend from $36 \mathrm{~m}$ to $2.7 \mathrm{~km}$. At the high declination of A2255 the array does not suffer from shadowing.

The pointing center of the telescope, as well as the phase center of the array, was directed towards RA $=17^{\mathrm{h}} 13^{\mathrm{m}} 00^{\mathrm{s}}$, Dec $=+64^{\circ} 07^{\prime} 59^{\prime \prime}$, which is the center of A2255. The time sampling is $30 \mathrm{~s}$ in the $25 \mathrm{~cm}$ and $85 \mathrm{~cm}$ datasets, and $10 \mathrm{~s}$ for the $2 \mathrm{~m}$ observations. This is generally sufficient to sample the phase fluctuations of the ionosphere and to avoid the smearing of sources at the outer edge of the field. During each observation, two pairs of calibrators, one polarized and one unpolarized, have been observed for $30 \mathrm{~min}$ each. Table 2 summarizes the observational parameters.

The technical details about the datasets will be discussed in the next sub sections. Here we give a short overview about the main steps taken for the data reduction. The data were processed with the WSRT-tailored NEWSTAR reduction package following mostly standard procedures: automatic interference flagging, self calibration, fast Fourier transform imaging, and CLEAN deconvolution (Högbom 1974). Further flagging based on the residual data after self calibration and model subtraction was done after each self calibration iteration. An on-line Hamming taper was used to lower the distant spectral side lobe level (Harris 1978). The final analysis was done using only the odd channels, because each Hamming tapered output channel is a linear combination of its direct neighbors and itself.

At $85 \mathrm{~cm}$ and $2 \mathrm{~m}$, the data were flux-calibrated using 3C 295, for which we adopted a flux of $63 \mathrm{Jy}$ and $95 \mathrm{Jy}$ at central frequencies of $346 \mathrm{MHz}$ and $148 \mathrm{MHz}$, respectively. At $25 \mathrm{~cm}$ the flux scales have been set using the flux calibrator CTD93, for which we adopted a flux of 5.2 Jy at $1220 \mathrm{MHz}$. The flux scale at $2 \mathrm{~m}$ is still uncertain to a level that we estimate of $5 \%$ and $1 \%$ at $25 \mathrm{~cm}$ and $85 \mathrm{~cm}$. We computed the flux densities of the calibrators across the entire bands assuming a spectral index of $\alpha=-0.5$ and $\alpha=-0.6$ for CTD93 and 3C 295 respectively (see Table 1).

\section{1. $25 \mathrm{~cm}$ dataset}

At $25 \mathrm{~cm}$, we observed A2255 for $4 \times 12 \mathrm{~h}$ with 4 different configurations of the WSRT. By stepping the 4 movable telescopes at $18 \mathrm{~m}$ increments, from $36 \mathrm{~m}$ to $90 \mathrm{~m}$, we pushed the grating lobe due to the regular $18 \mathrm{~m}$ baseline increment to a radius of $\sim 1^{\circ}$. 
Table 2. Observations overview.

\begin{tabular}{|c|c|c|c|c|c|c|}
\hline Wavelength & $\begin{array}{c}\text { Frequency range } \\
(\mathrm{MHz})\end{array}$ & Calibrators & Observation ID & $\begin{array}{l}\text { RT9-RTA } \\
\text { (m) }\end{array}$ & Start date (UTC) & End date (UTC) \\
\hline $25 \mathrm{~cm}$ & $1159-1298 \mathrm{MHz}$ & $\begin{array}{c}\text { 3C286, CTD93 } \\
3 \mathrm{C} 147,3 \mathrm{C} 138\end{array}$ & $\begin{array}{l}10702691 \\
10702898 \\
10702958 \\
10703066\end{array}$ & $\begin{array}{l}36 \\
54 \\
72 \\
90\end{array}$ & $\begin{array}{ll}2007 / 06 / 18 & 17: 00: 15 \\
2007 / 06 / 28 & 16: 20: 55 \\
2007 / 07 / 03 ~ 16: 01: 15 \\
2007 / 07 / 10 & 15: 33: 45\end{array}$ & $\begin{array}{ll}2007 / 06 / 19 & 04: 58: 50 \\
2007 / 06 / 29 & 04: 19: 25 \\
2007 / 07 / 04 & 03: 59: 45 \\
2007 / 07 / 11 & 03: 10: 35\end{array}$ \\
\hline $85 \mathrm{~cm}$ & $310-380 \mathrm{MHz}$ & $\begin{array}{c}\text { DA240, 3C 295, } \\
\text { PSR1937+21, } \\
\text { 3C48 }\end{array}$ & $\begin{array}{l}10602227 \\
10602239 \\
10602259 \\
10602224 \\
10602372 \\
10602340\end{array}$ & $\begin{array}{l}36 \\
48 \\
60 \\
72 \\
84 \\
96\end{array}$ & $\begin{array}{l}2006 / 05 / 09 \text { 19:36:20 } \\
\text { 2006/05/10 19:32:20 } \\
\text { 2006/05/11 19:28:20 } \\
\text { 2006/05/16 19:08:40 } \\
\text { 2006/05/19 18:57:00 } \\
2006 / 05 / 22 \text { 18:45:10 }\end{array}$ & $\begin{array}{ll}2006 / 05 / 10 & 07: 35: 20 \\
2006 / 05 / 10 & 07: 31: 20 \\
2006 / 05 / 12 & 07: 27: 20 \\
2006 / 05 / 17 & 07: 07: 40 \\
2006 / 05 / 20 & 06: 56: 00 \\
2006 / 05 / 23 & 06: 44: 10\end{array}$ \\
\hline $2 \mathrm{~m}$ & $115-175 \mathrm{MHz}$ & $\begin{array}{c}\text { DA240, 3C 295, } \\
\text { PSR1937+21, } \\
\text { 3C48 }\end{array}$ & $\begin{array}{l}10702727 \\
10703226 \\
10703186 \\
10703004 \\
10703056 \\
10703099\end{array}$ & $\begin{array}{l}36 \\
48 \\
60 \\
72 \\
84 \\
96\end{array}$ & $\begin{array}{ll}2007 / 06 / 21 & 16: 48: 15 \\
2007 / 07 / 19 & 14: 58: 05 \\
2007 / 07 / 17 & 15: 05: 55 \\
2007 / 07 / 05 & 15: 53: 15 \\
2007 / 07 / 09 & 15: 37: 25 \\
2007 / 07 / 12 & 15: 25: 35\end{array}$ & $\begin{array}{ll}2007 / 06 / 22 & 04: 47: 05 \\
2007 / 07 / 20 & 02: 56: 55 \\
2007 / 07 / 18 & 03: 04: 45 \\
2007 / 07 / 06 & 03: 52: 05 \\
2007 / 07 / 10 & 03: 36: 15 \\
2007 / 07 / 13 & 03: 24: 25\end{array}$ \\
\hline
\end{tabular}

At this wavelength, the receiving band is divided into 8 contiguous slightly overlapping sub-bands of $20 \mathrm{MHz}$ centered at 1169, 1186, 1203, 1220, 1237, 1254, 1271, and $1288 \mathrm{MHz}$. Each sub-band is covered by 64 channels in 4 cross-correlations to recover all Stokes parameters.

Because of limitations of the software in handling files larger than $2.15 \mathrm{~GB}$, the data reduction was done for each frequency band independently. Thus, the original dataset has been split in 8 sub-datasets, each containing the $4 \times 12$ h of data, but just in one frequency band.

Because of strong RFI, 2 out of the original 8 frequency subbands have not been used for imaging. Moreover, the presence of a strong off-axis source caused phase errors affecting the central field of the final image. The peeling procedure that we used to solve for those problems was successful only in the lower 3 frequency bands, where the problematic source is brighter (see Sect. 2.2). Therefore, the total amount of data that we could use for imaging was $\sim 35 \%$. At $25 \mathrm{~cm}$, the resolution is $14^{\prime \prime} \times 15^{\prime \prime}$.

\subsection{Off-axis errors in $25 \mathrm{~cm}$ maps}

High dynamic range imaging is seriously limited by the phase stability of the atmosphere (troposphere and ionosphere), which causes the presence of spiky patterns surrounding the brightest off-axis sources within the field of view of the interferometer. This pattern is due to the instantaneous fan beam response of the WSRT, which rotates clockwise from position angle $+90^{\circ}$ to $+270^{\circ}$ during the $12 \mathrm{~h}$ synthesis time.

The selfcal algorithm tries to minimize the time variations between data and the model of the sky, applying only one averaged time-dependent correction in the $u v$ plane. If in the field of view there are bright off-axis sources that seem to move during the observation, due to differential tropospheric refraction or rapid ionospheric phase instabilities, the selfcal will not be able to correct simultaneously for the phase errors associated with them. Thus, the final map will show artefacts around the sources, as secondary lobes, or in the source itself, which will appear distorted. The only way to remove the off-axis errors in the maps is to "peel" each problematic source out of the dataset, using its own direction-dependent selfcal corrections. This technique has been recently developed to solve for the ionospheric phase disturbances in low-frequency observations (e.g. Cotton et al. 2004; Intema 2007), but it can be also applied at higher frequencies, when phase errors due to the differential tropospheric effects are also present in high dynamic range images.

The peeling scheme involves self calibration on individual bright sources. It produces phase corrections per array element for several viewing directions, repeatedly using the following recipe:

- subtraction of all but the brightest source from UV data, using the best model and calibration available;

- several rounds of phase only self calibration and imaging on the brightest source;

- subtraction of the brightest source from the original UV data, using model and calibration from the previous step.

We applied this algorithm to the $25 \mathrm{~cm}$ dataset, which is the only one affected by off axis errors that affect the analysis at the center of the maps. Also our maps at $85 \mathrm{~cm}$ and at $2 \mathrm{~m}$ showed off axis errors, but they were confined in areas far away from the field center. Therefore no peeling procedure was needed at these wavelengths. At $25 \mathrm{~cm}$ the phase errors were associated with the off axis source $4 \mathrm{C}+64.21$, located at $\mathrm{RA}=17^{\mathrm{h}} 19^{\mathrm{m}} 59^{\mathrm{s}}$, Dec $=+64^{\circ} 04^{\prime} 40^{\prime \prime}$. In Fig. 1, we show the result of the procedure. Without peeling, $4 \mathrm{C}+64.21$ shows a distorted shape, with a positive spike going towards east and a long negative spike directed towards the opposite direction, where the center of the cluster is located. The source is also surrounded by ring structures, which were left after the deconvolution. After peeling, the source is basically removed. The flux below which the sources have been subtracted for this procedure is $\sim 1 \mathrm{Jy}$.

The peeling procedure has been applied in each of the 6 frequency bands we could use at $25 \mathrm{~cm}$ (Sect. 2.1). Unfortunately, it turned out to be satisfactory just for the three lower frequency bands, where the selfcal corrections for the steep spectrum $4 \mathrm{C}+64.21$ can be better determined, given the higher signal to noise ratio of the data. Thus, we decided to use only these 3 frequency bands to make the final image for the further analysis. 

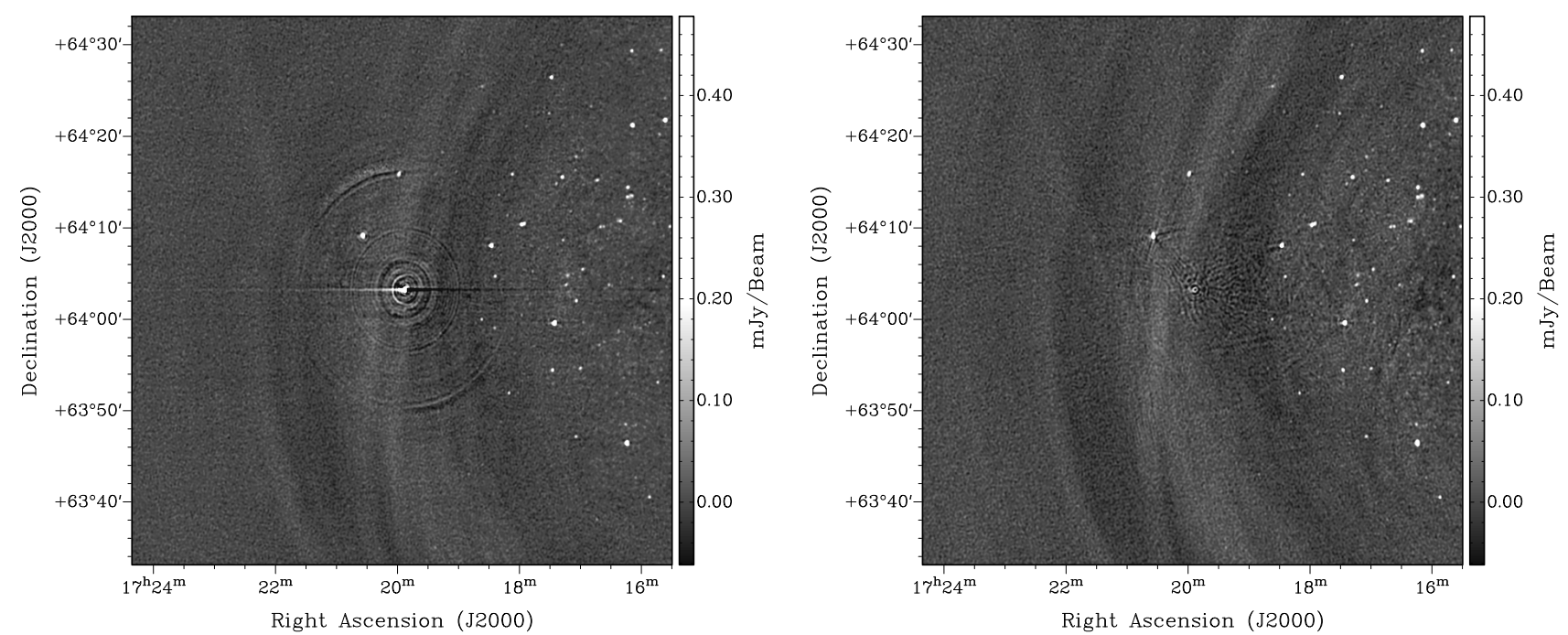

Fig. 1. Grey scale images of $4 \mathrm{C}+64.21$ at $25 \mathrm{~cm}$ before (left panel) and after (right panel) applying the peeling procedure. The resolution is $14^{\prime \prime} \times 15^{\prime \prime}$. Left panel: the source shows phase-related errors, like ring structures and a negative and a positive spike along the east-west direction. Right panel: just a small residual is left at the source location.

\section{3. $85 \mathrm{~cm}$ dataset}

To fully image the primary beam, six array configurations were used for the observations, with the four movable telescopes stepped at $12 \mathrm{~m}$ increments (i.e. half the dish diameter) and the shortest spacing running from $36 \mathrm{~m}$ to $96 \mathrm{~m}$. This provides continuous uv-coverage with interferometer baselines ranging from $36 \mathrm{~m}$ to $2760 \mathrm{~m}$.

The receiving band is covered by 8 sub-bands of $10 \mathrm{MHz}$ centered at 315, 324, 332, 341, 350, 359, 367, and $376 \mathrm{MHz}$. Each sub-band is covered by 128 channels in 4 cross-correlations to recover all Stokes parameters. The $85 \mathrm{~cm}$ dataset was split in 16 sub-datasets, each containing $6 \times 12 \mathrm{~h}$ of observation, but in one frequency half-band only.

The imaging was done using 5 out of the 6 nights of observation, because of the poor phase stability of the ionosphere during the third night (RT9-RTA $=60 \mathrm{~m}$ ). The total amount of flagged data was $\sim 25 \%$. At $85 \mathrm{~cm}$ wavelength, the resolution is $54^{\prime \prime} \times 64^{\prime \prime}$.

\subsection{2 m dataset (LFFE-band observations)}

At $2 \mathrm{~m}, \mathrm{~A} 2255$ was observed for $6 \times 12 \mathrm{~h}$ to fully image the primary beam.

The LFFE (Low-Frequency Front End) at the WSRT is covered by 8 frequency sub-bands of $2.5 \mathrm{MHz}$ centered at 116,121 , $129,139,141,146,156$, and $162 \mathrm{MHz}$. Each sub-band is divided in 512 channels.

Given the large size of the final dataset ( 250 GB) and the limitations in the maximum size of a NEWSTAR dataset, the observations have been split in 240 sub-datasets, each containing data for a single night of observation and with a limited number of channels. Therefore, for a single spacing and for each subband, we worked with 5 datasets. To produce the final map, we processed the 240 sub datasets separately, self calibrating them with different models and doing the imaging and the deconvolution steps 240 times. We did not obtain a good image through the normal selfcal procedure. The best image has been obtained using a simplified self calibration where we only solved for the phase slope across the array. In this case, only the phases were self calibrated. At the end, the images were combined, weighting them for the input number of visibilities.

Due to serious problems with the correlators of the WSRT during the night between the 17th July and the 18th July 2007 $($ RT9-RTA $=60 \mathrm{~m})$, the final data reduction was done using $5 \times 12 \mathrm{~h}$ of observation. Moreover, given serious ionospheric instabilities during the last night of observations (RT9-RTA = $96 \mathrm{~m}$ ), we decided to not include this 12 hours run in imaging. The total amount of flagged data was $\sim 35 \%$. At $2 \mathrm{~m}$, the resolution is $163^{\prime \prime} \times 181^{\prime \prime}$.

\subsection{A few issues related to low-frequency observations}

The low-frequency radio sky is very bright and populated by strong radio sources, such as Cas A, Cyg A, Vir A and Tau A, whose flux densities vary from 1000-10000 Jy. Even far from the field center, the (relatively) high distant sidelobe levels of the primary beam ("only" -30 to $-40 \mathrm{~dB}$ ) keeps these sources very bright, giving rise to significant side lobes in the final images. Solar flares can also affect the data, causing interferences in the short baselines and/or grating rings in the imaged central field.

During the editing and imaging processes at low frequency, we had to take care of Cas A, Cyg A and the Sun. Cas A lies at a projected distance of $\sim 40^{\circ}$ from the cluster, Cyg A at $\sim 30^{\circ}$ and the Sun at $\sim 85^{\circ}$. At $85 \mathrm{~cm}$ and at $2 \mathrm{~m}$, we used models of Cas A and Cyg A and we subtracted them from the data during imaging. To remove the interferences generated by the Sun in the $85 \mathrm{~cm}$ dataset, we flagged the short baselines in the hour angles in which the Sun was above the horizon $\left(+30^{\circ} \leq \mathrm{HA} \leq\right.$ $\left.+90^{\circ}\right)$. At $2 \mathrm{~m}$, the Sun was high in the sky for most part of the observation. In this case, we produced different cleaning models of this source for the different nights and we subtracted them during the final imaging.

\subsection{Flux scale at $2 m$ wavelength}

The flux scale at low frequencies is not very accurately determined. Until a more definitive flux scale is in place (this is being developed for LOFAR) we use the radio source 3C 295 as the primary WSRT flux calibrator at low frequencies. We have adopted a flux density at $150 \mathrm{MHz}$ of $95 \mathrm{Jy}$ for 3C 295 
Table 3. Parameters of the observations and of the final full resolution maps.

\begin{tabular}{ccccccc}
\hline \hline Wavelength & $\begin{array}{c}\text { Full resolution } \\
\prime\end{array}$ & $\begin{array}{c}\text { Largest detectable structure } \\
,\end{array}$ & $\sigma_{\mathrm{F}}{ }^{a}$ & $\begin{array}{c}\sigma_{\text {th }}{ }^{b} \\
\text { mJy/beam }\end{array}$ & $\begin{array}{c}\sigma_{\text {confcenter }}{ }^{c} \\
\text { mJy/beam }\end{array}$ & $\begin{array}{c}\sigma_{\text {obscenter }}{ }^{d} \\
\text { mJy/beam }\end{array}$ \\
\hline $2 \mathrm{~m}$ & $163 \times 181$ & 191 & $5 \%$ & 0.85 & 3 & 3.1 \\
\hline $85 \mathrm{~cm}$ & $54 \times 64$ & 81 & $1 \%$ & 0.05 & 0.25 & 0.25 \\
\hline $25 \mathrm{~cm}$ & $14 \times 15$ & 24 & $1 \%$ & 0.010 & 0.008 & 0.012 \\
\hline
\end{tabular}

${ }^{a}$ Flux scale uncertainty.

${ }^{b}$ Thermal noise, constant over the maps.

${ }^{c}$ Confusion noise.

${ }^{d}$ Observed noise at the pointing position.

and a power-law spectral index of -0.6 in the frequency range from 115-175 MHz. We believe this number to be accurate to about $5 \%$.

A major component of the system noise (receiver + sky) at low frequencies is due to our Galaxy. The System Equivalent Flux Density (SEFD) of the telescopes at $150 \mathrm{MHz}$ is about $8000 \mathrm{Jy}$ in the Galactic areas where 3C 295 happens to be located, but it rises to well over $10000 \mathrm{Jy}$ in the Galactic plane. Because the WSRT receivers operate with an automatic gain control (AGC) system before the analog-to-digital converter, it continuously measures the total power to allow corrections for the variable input levels. Unfortunately, most of the time the total powers detectors (which integrate the power over the whole 2.5 MHz sub-band) are corrupted by RFI so we can not automatically correct the correlation coefficients for the variations in system noise. The LFFE band is full of mostly impulsive and narrow band RFI coming from airplanes, satellites, and mobile users as well as electronic hardware within the building which is located halfway the array. At the high spectral resolution $(10 \mathrm{kHz})$ provided by the backend most of this RFI can be excised. However, the total power data must be manually inspected for suitable stretches of power level measurements. These data form the basis for a manual correction of the flux scale.

For A2255 the total power ratio between the cluster region and the 3C 295 field is $1.15 \pm 0.05$ at $141 \mathrm{MHz}$. Following the transfer of the complex gain correction determined for 3C 295 we have therefore applied an additional correction of a factor 1.15 to the visibility data.

\subsection{Errors and noise in the final images}

Knowing the value of the error in an image is essential in determining the reliability of the image and of the derived parameters. The observed errors $\left(\sigma_{\mathrm{obs}}\right)$ in a map mainly consist of:

- the thermal noise $\left(\sigma_{\text {th }}\right)$, which is due to the stochastic errors, coming from the Galaxy, receivers and other electronics used in the array;

- the confusion noise $\left(\sigma_{\text {conf }}\right)$, which actually consists of three contributions:

1. the "normal" sidelobe noise, which arises from the sum of all the sidelobes responses to the very large number of sources visible within the field of view. This noise can be minimized by deconvolution;

2. the "classical" source confusion, which is associated with the number of sources within the same beam;

3. the "error" sidelobe noise, which results from calibration errors and non-isoplanaticity.

It is important to appreciate the difference between thermal and confusion noise. While the former is different every time we observe a source, the latter is always the same for a given PSF, i.e. it repeats itself every time we observe the same field.

In Table 3, we list the estimated $\sigma_{\text {th }}$ and $\sigma_{\text {conf }}$ for the three observing wavelengths. Reliable estimates of the thermal noise have been obtained from polarization images $(Q, U, V)$ and narrow spectral bands. The confusion limit for WSRT at $21 \mathrm{~cm}$ was determined by Garrett et al. (2000) to be $\sim 5 \mu \mathrm{Jy}$. Because the PSF of the WSRT depends on the declination, this value refers to Dec $=+6^{\circ}$. At a different wavelength $\left(\lambda_{2}\right)$ it is given by

$\sigma_{\lambda_{2}}=\sigma_{\lambda_{1}}\left(\frac{\lambda_{2}}{\lambda_{1}}\right)^{2.75}$

where $\sigma_{\lambda_{1}}$ is the confusion limit at $\lambda_{1}=21 \mathrm{~cm}$ and the exponent $(-2.75)$ takes into account the spectral index dependence $(-0.75)$ and the different synthesized beam $(-2)$.

Assuming that $\sigma_{\text {th }}$ and $\sigma_{\text {conf }}$ are uncorrelated, the observed noise in the final map as a function of the distance from the center of the field is given by:

$\sigma_{\text {obs }}(r)=\sqrt{\sigma_{\text {th }}^{2}+\sigma_{\text {conf }}^{2}(r)}$.

In Fig. 2, we plot the observed noise levels, uncorrected for the primary beam, as a function of the distance from the field center for the final $2 \mathrm{~m}, 85 \mathrm{~cm}$, and $25 \mathrm{~cm}$ full resolution maps. Because the classical confusion noise will be attenuated by the primary beam, the observed noise decreases from the center to the edges of the field, where it approaches the thermal noise level. This trend, more clear in the low-frequency maps, is a problem when trying to assess the significance of the central extended features in the contour maps. In principle, we should consider as detection limit the noise observed at the center of the field and plot the contours starting from, for example, 3 times this value. However, it is worth noting that in this area we are confusion limited (this is a situation similar to that found in deep optical images of galaxies, where the central fluctuations are often dominated by the large number of stars within a resolution element). Therefore, in order to show the significance of the features in our final contour maps, we start plotting the contours from 3 times the noise observed at the edge of the fully imaged field. To assess the significance of the detected structures with respect to the background fluctuations, we advice the reader to compare the contour maps at $25 \mathrm{~cm}, 85 \mathrm{~cm}$, and $2 \mathrm{~m}$ with their grey scale version in Fig. 3.

\subsection{Flux measurement uncertainties}

Because of the errors present in the final images, the flux and spectral index estimates are affected by uncertainties. The error associated with the flux depends on:

- the observed noise in the final maps $\left(\sigma_{\mathrm{obs}}\right)$; 

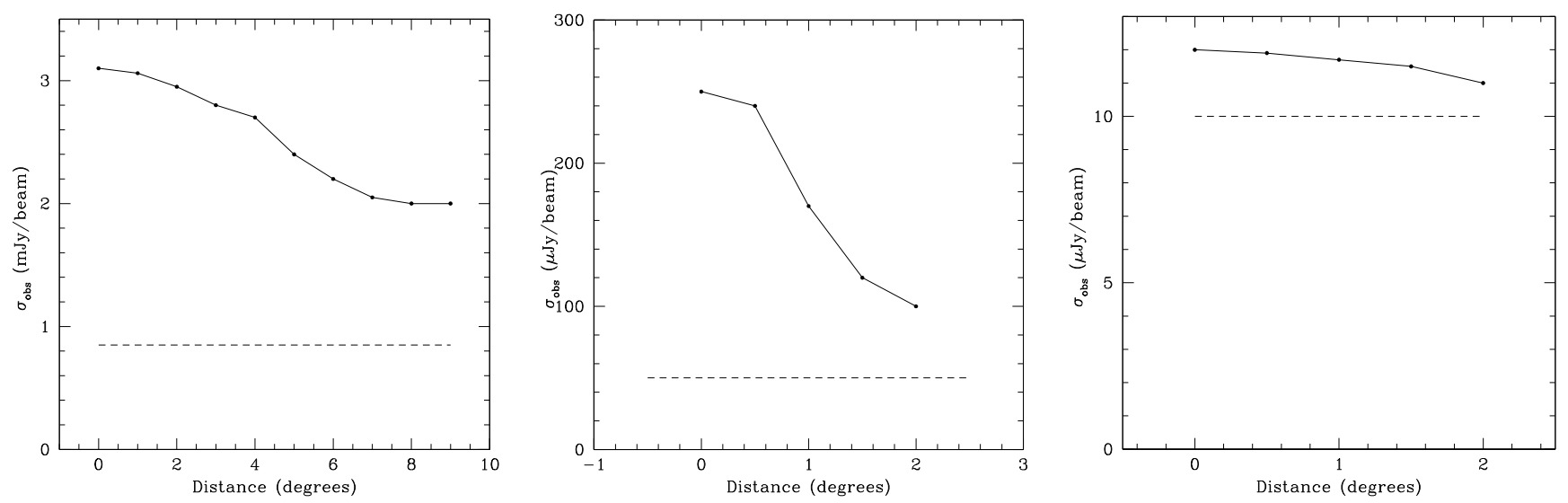

Fig. 2. Observed noise level uncorrected for the primary beam as a function of distance from the field center for the $2 \mathrm{~m}$ (left panel), $85 \mathrm{~cm}$ (middle panel), and $25 \mathrm{~cm}$ (right panel) maps. The dashed line represents the estimated thermal noise level, again uncorrected for the primary beam.

- the error due to the negative bowl, which arises around the extended structures because of the missing short spacings $\left(\sigma_{\text {bowl }}\right)$. This severely affects the non full resolution maps only (see Sect. 2.9). In our case, its value was estimated by determining the mean value of the negative bowl around the central maximum in the antenna pattern;

- the uncertainty related with the flux of the calibrator $\left(\sigma_{\mathrm{c}}\right)$. This is a scale error which apply to the whole map.

Under the assumption that these three uncertainties are uncorrelated, the final error on the flux is

$\sigma_{\mathrm{F}}=\sqrt{\sigma_{\mathrm{c}}^{2}+\sigma_{\mathrm{obs}}^{2}+\sigma_{\text {bowl }}^{2}}$.

The uncertainty on the spectral index is given by

$\sigma_{\alpha}=\frac{1}{\ln \left(v_{2} / v_{1}\right)} \sqrt{\left(\frac{\sigma_{\mathrm{F} 1}}{F_{1}}\right)^{2}+\left(\frac{\sigma_{\mathrm{F} 2}}{F_{2}}\right)^{2}}$,

where $F_{1} \pm \sigma_{\mathrm{F} 1}$ and $F_{2} \pm \sigma_{\mathrm{F} 2}$ are the flux densities and the corresponding errors at frequency $v_{1}$ and $v_{2}$, respectively.

\subsection{Making maps at $25 \mathrm{~cm}, 85 \mathrm{~cm}$, and $2 \mathrm{~m}$ for a spectral index analysis}

To make the final spectral index maps, we tapered the data to the same low resolution of the $2 \mathrm{~m}$ dataset $\left(163^{\prime \prime} \times 181^{\prime \prime}\right)$, and we cut

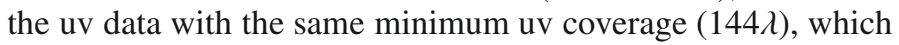
is determined by the $25 \mathrm{~cm}$ data.

The missing short spacings in the low resolution $2 \mathrm{~m}$ and $85 \mathrm{~cm}$ maps created a negative bowl around the central radio halo. To remove as much as possible of the negative bowl, we cleaned very deep the halo emission. At $2 \mathrm{~m}$, the final map was obtained including in imaging only the three frequency bands least affected by RFI. Finally, the maps have been corrected for the total power primary beam of the WSRT, which can be approximated by

$G(v, r)=\cos ^{6}(\mathrm{c} v r)$,

where $c$ is a constant $\sim 0.064, v$ is the observing frequency in $\mathrm{MHz}$ and $r$ is the radius from the pointing center in degrees.

\section{Results}

A grey scale version of the final full resolution maps at $25 \mathrm{~cm}$, $85 \mathrm{~cm}$ and $2 \mathrm{~m}$ is shown in Fig. 3. In the following subsections, we describe the features detected at each wavelength.

\section{1. $25 \mathrm{~cm}$ map}

The $25 \mathrm{~cm}$ map is presented in Fig. 4. The image, which has a noise level of $11 \mu \mathrm{Jy}$ at the edge of the field, shows the well known extended halo, located at the cluster center, the relic, that lies $10^{\prime}$ to the northeast from it, and 3 extended radio galaxies at a very large distance from the center of the cluster: the Embryo and the Beaver lie at $\sim 1.6 \mathrm{Mpc}$ from the cluster center, while the Bean lies at more than $3.5 \mathrm{Mpc}$ (quoted names are taken from Harris et al. 1980). Since more relic features are detected at low frequency around A2255 (Sect. 3.2), from now on we will refer to the relic as NE (north-east) relic. Zooming into the central region of A2255, we notice the presence of 4 additional extended cluster radio galaxies: the Goldfish, the Double, the original TRG, and the Sidekick. The positions of the 7 radio galaxies are listed in Table 4. Each of them has an optical counterpart that belongs to the cluster (Miller \& Owen 2003).

The halo has a rectangular shape and shows a filamentary structure, which is in agreement with previous $21 \mathrm{~cm}$ VLA observations (Govoni et al. 2005). Given the high sensitivity of our new observations, halo and NE relic look more extended and directly connected by a radio bridge, which extends towards the north-east and seems associated with two features; the former is located at RA $=17^{\mathrm{h}} 13^{\mathrm{m}} 13^{\mathrm{s}}$, Dec $=+64^{\circ} 12^{\prime} 54^{\prime \prime}$ and belongs to the NE relic, the latter lies at RA $=17^{\mathrm{h}} 14^{\mathrm{m}} 18^{\mathrm{s}}$, $\mathrm{Dec}=+64^{\circ} 16^{\prime} 10^{\prime \prime}$. They are labeled respectively $\mathrm{C} 1$ and $\mathrm{C} 2$ in Fig. 4. The physical parameters of halo and NE relic are reported in Table 5.

We detect a very low surface brightness feature at location $\mathrm{RA}=17^{\mathrm{h}} 12^{\mathrm{m}} 12^{\mathrm{s}}$, Dec $=+64^{\circ} 30^{\prime} 08^{\prime \prime}$, that is associated with one of the two filaments of the NW relic detected at $85 \mathrm{~cm}$ (Sect. 3.2). Since it lies in a pretty empty region of the radio sky, a possible association of this feature with a collection of point sources or with a central radio galaxy seems unlikely.

\section{2. $85 \mathrm{~cm}$ map}

The $85 \mathrm{~cm}$ map of A2255 is presented in Fig. 6. With noise levels ranging between $0.08 \mathrm{mJy} /$ beam to $0.25 \mathrm{mJy} / \mathrm{beam}$, limited by classical confusion noise (see Sect. 2.7 and Table 3), it improves over previous imaging at close wavelength (Feretti et al. 1997) by a factor of 20. The overlay of the radio map with the red band Digitized Sky Survey (DSS) optical image is presented in Fig. 5. This image clearly shows the extension of the radio emission compared to the optical galaxies. 

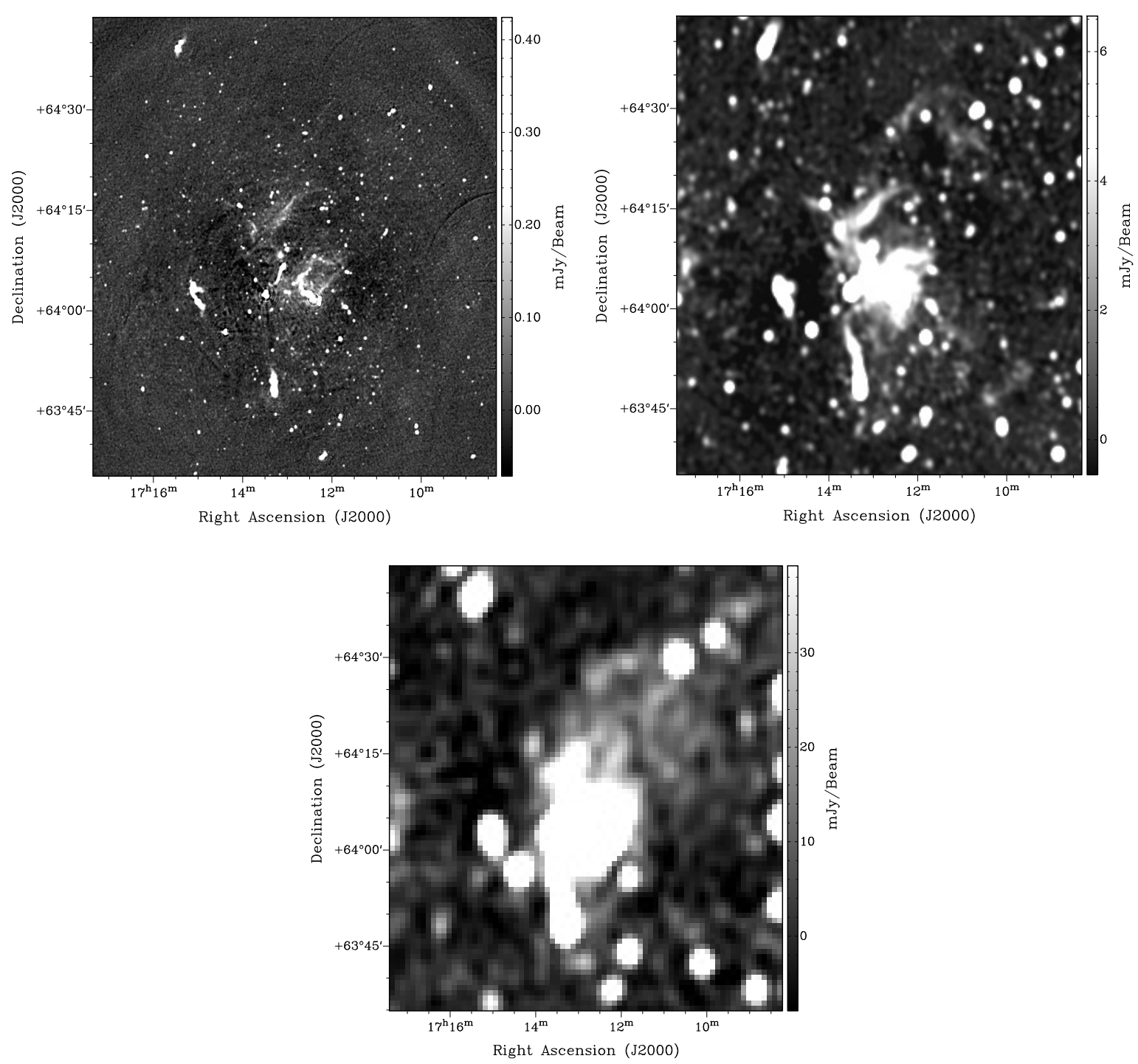

Fig. 3. Grey scale images of A2255 at $25 \mathrm{~cm}$ (top left panel), $85 \mathrm{~cm}$ (top right panel), and $2 \mathrm{~m}$ (bottom panel). All images cover the same area of the sky. The resolutions are $14^{\prime \prime} \times 15^{\prime \prime}(25 \mathrm{~cm}), 54^{\prime \prime} \times 64^{\prime \prime}($ at $85 \mathrm{~cm})$ and $163^{\prime \prime} \times 181^{\prime \prime}$ (at $\left.2 \mathrm{~m}\right)$. The maps are not corrected for the primary beam.

The halo, the NE relic, and the radio galaxies belonging to the cluster are detected. The central radio halo looks much more complex than in previous images at the same frequency (Feretti et al. 1997) and it is more extended than at $25 \mathrm{~cm}$, in particular towards the S and SW. We notice that the southern region of the halo is directly connected, in projection, to the tail of the Beaver radio galaxy, which has doubled its length to almost $1 \mathrm{Mpc}$ between $25 \mathrm{~cm}$ and $85 \mathrm{~cm}$. Feature C2 in the $25 \mathrm{~cm}$ map (see Fig. 4) is now more prominent and it looks directly associated to the NE relic. The physical parameters of halo and NE relic at $85 \mathrm{~cm}$ are listed in Table 5.

The high sensitivity of our observations allow us to detect two new extended features at a projected distance of $2 \mathrm{Mpc}$ from the cluster center. The new "relics" are located NW and SW of the center of the cluster and previous $21 \mathrm{~cm}$ images of A2255 revealed that they are genuine features and not a collection of discrete sources (Pizzo \& de Bruyn 2008). From now on, we will call them the NW (north-west) and SW (south-west) relic, respectively. They have different shapes. The SW relic appears like a filament of about $8^{\prime}$ in length and $2^{\prime}$ in width. It has the same orientation of the known NE relic, but is located on the opposite side from the cluster center and at a double distance from it. The
NW relic has a more complex morphology. We can distinguish 2 filaments, labeled NW1 and NW2 in Fig. 6. NW1 points towards the cluster center and has a length of $\sim 8^{\prime} \times 1^{\prime}$, while NW2 is $\sim 13^{\prime} \times 1^{\prime}$ and is perpendicular to NW1. The SW and NW relics have integrated flux densities of $\sim 17 \mathrm{mJy}$ and $\sim 61 \mathrm{mJy}$, respectively. The physical properties of the newly detected structures and their origin have been investigated by Pizzo et al. (2008), who suggested a connection with LSS shocks.

Other extended diffuse low surface brightness features are detected to the east $\left(\mathrm{RA}=17^{\mathrm{h}} 16^{\mathrm{m}} 57^{\mathrm{s}}\right.$, Dec $\left.=+64^{\circ} 18^{\prime} 45^{\prime \prime}\right)$ and to the west $\left(\mathrm{RA}=17^{\mathrm{h}} 07^{\mathrm{m}} 08^{\mathrm{s}}\right.$, Dec $\left.=+63^{\circ} 59^{\prime} 36^{\prime \prime}\right)$ of the cluster center. Their nature is still unclear. Moreover, the map shows positive and negative fluctuations on a scale of 0.5 to 1 degree, which are likely due to our Galaxy.

\section{3. $2 m$ map}

The $2 \mathrm{~m}$ map is shown in Fig. 7. The noise ranges between $2 \mathrm{mJy}$ and $3 \mathrm{mJy}$ and is limited by classical confusion noise in the inner part of the map (see Sect. 2.7 and Table 3).

Around the central radio halo, the known radio galaxies belonging to the cluster are still detected. The diffuse emission 


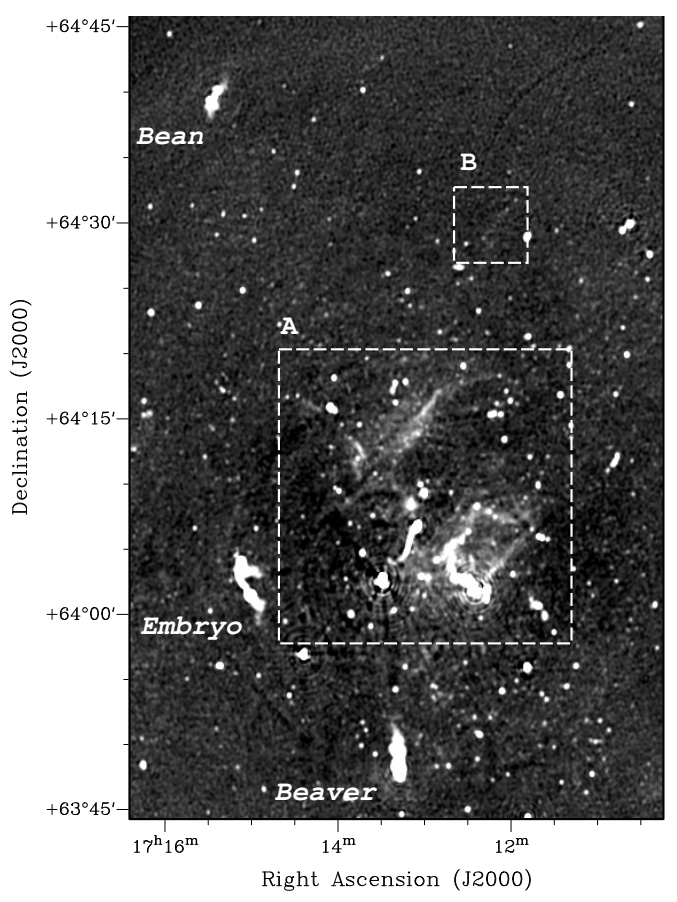

A

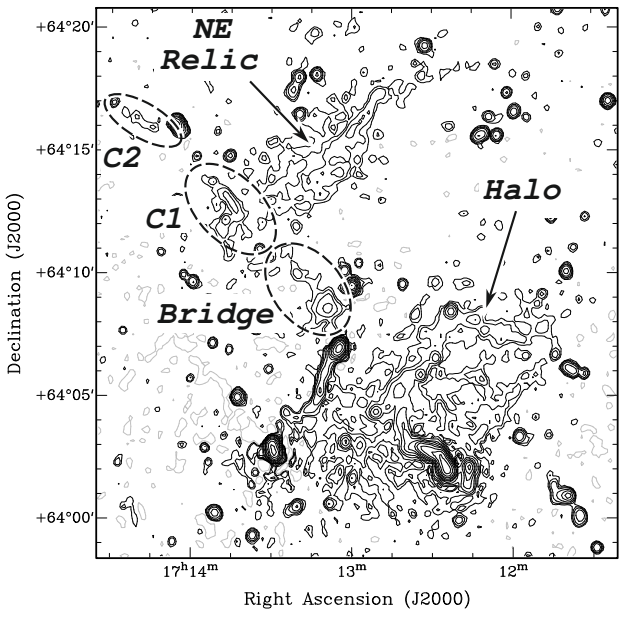

B

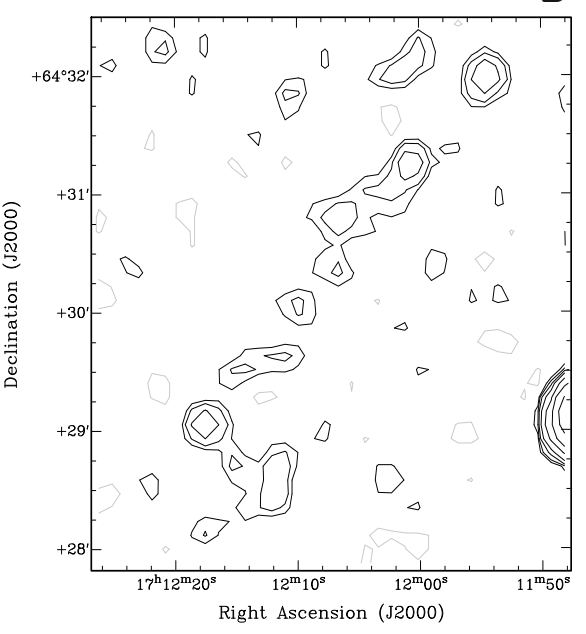

Fig. 4. Top panel: big field grey scale image of A2255 at $25 \mathrm{~cm}$. The radio galaxies Bean, Embryo, and Beaver are visible, at large distance from the cluster center. The resolution is $14^{\prime \prime} \times 15^{\prime \prime}$. The noise level at the edge of the fully imaged field is $11 \mu \mathrm{Jy} / \mathrm{beam}$ (see Fig. 2). Bottom left panel: zoom into the central cluster region. We can distinguish halo, NE relic, bridge connection between them and the radio galaxies Goldfish, Double, The original TRG, and Sidekick. C1 and C2 seem to be an extension of the radio bridge (see text). The contours are $-0.03,0.03,0.06,0.12,0.24$, $0.48,0.96,2,4,8,16,32 \mathrm{mJy} /$ beam. Bottom right panel: zoom into the region of the cluster where the new $\mathrm{NW}$ relic has been detected at $85 \mathrm{~cm}$ (Sect. 3.2). At $25 \mathrm{~cm}$, the only filament pointing towards the cluster center (NW1) is detected, but at very low level $(1 \sigma)$. Here the contours are -0.01 (grey), $0.01,0.02,0.04,0.06,0.08,0.16,0.32,0.64,1.2,2.4 \mathrm{mJy} /$ beam. The maps are not corrected for the primary beam.

Table 4. The extended radio galaxies of A2255.

\begin{tabular}{ccc}
\hline \hline Name & RA $(\mathrm{J} 2000)$ & Dec $(\mathrm{J} 2000)$ \\
\hline Bean & $17^{\mathrm{h}} 15^{\mathrm{m}} 31^{\mathrm{s}}$ & $+64^{\circ} 39^{\prime} 28^{\prime \prime}$ \\
Beaver & $17^{\mathrm{h}} 13^{\mathrm{m}} 19^{\mathrm{s}}$ & $+63^{\circ} 48^{\prime} 16^{\prime \prime}$ \\
Double & $17^{\mathrm{h}} 13^{\mathrm{m}} 28^{\mathrm{s}}$ & $+64^{\circ} 02^{\prime} 50^{\prime \prime}$ \\
Embryo & $17^{\mathrm{h}} 15^{\mathrm{m}} 05^{\mathrm{s}}$ & $+64^{\circ} 03^{\prime} 42^{\prime \prime}$ \\
Goldfish & $17^{\mathrm{h}} 13^{\mathrm{m}} 04^{\mathrm{s}}$ & $+64^{\circ} 06^{\prime} 56^{\prime \prime}$ \\
TRG & $17^{\mathrm{h}} 12^{\mathrm{m}} 23^{\mathrm{s}}$ & $+64^{\circ} 01^{\prime} 46^{\prime \prime}$ \\
Sidekick & $17^{\mathrm{h}} 12^{\mathrm{m}} 16^{\mathrm{s}}$ & $+64^{\circ} 02^{\prime} 14^{\prime \prime}$ \\
\hline
\end{tabular}

associated with A2255 seems to be rather complex. The radio halo is extended towards NW and is connected to the NW relic.
The largest detectable structure in our $85 \mathrm{~cm}$ observations is $\sim 1^{\circ}$, which means that non detecting this extended feature at $85 \mathrm{~cm}$ cannot be due to uv plane coverage issues. Instead, it supports the more likely hypothesis that the new emission region is a steep spectrum feature, whose nature is different from the NW relic. We note that point sources might make an important contribution to the new extended emission feature. To test its nature, we removed the contribution of point sources detected at $25 \mathrm{~cm}$ in this area, assuming a nominal spectral index of $\alpha=-0.7$. The total flux subtracted in the region is $635 \mathrm{mJy}$. The result, shown in Fig. 8, confirms the genuine diffuse nature of the feature. The source at location RA $=17^{\mathrm{h}} 10^{\mathrm{m}} 37^{\mathrm{s}}$, Dec $=+64^{\circ} 30^{\prime} 24^{\prime \prime}$ is due to a blend of 2 point sources, as can be seen at higher frequency (Fig. 6). Given their steep spectrum $(\alpha \sim-1)$, they are 

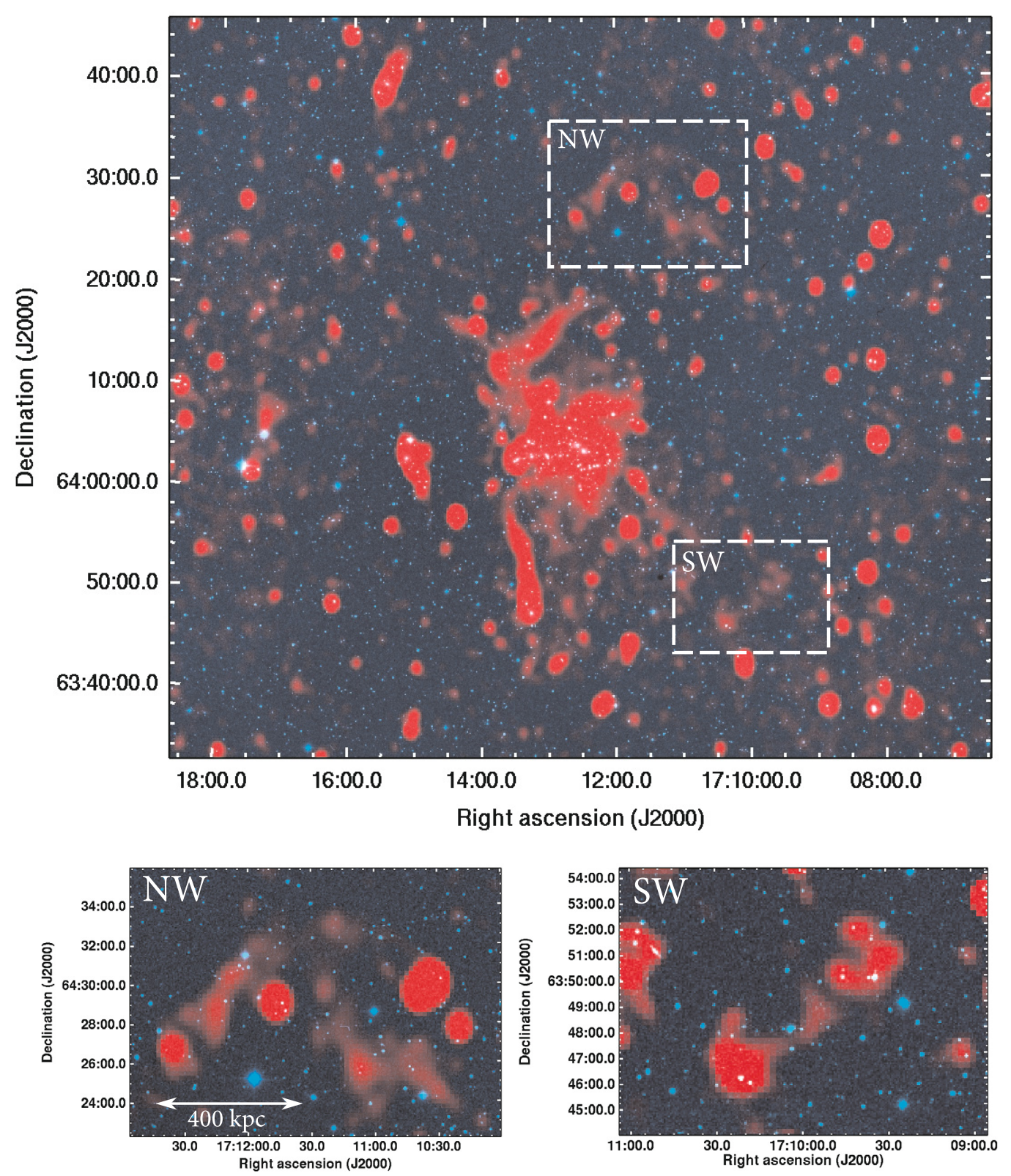

Fig. 5. Composite images of A2255 obtained from superposing the radio and optical images. The WSRT $85 \mathrm{~cm}$ radio map (in grey) for the total field (central panel), NW relic (bottom left panel), and SW relic (bottom right panel) are shown overlaid on the red band Digitized Sky Survey image (black and white). The radio image has a resolution of $54^{\prime \prime} \times 64^{\prime \prime}$ and it is not corrected for the primary beam.

still visible after the subtraction. We computed the upper limit for the spectral index of this feature using maps at $2 \mathrm{~m}$ and at $85 \mathrm{~cm}$ restored with the same resolution $\left(163^{\prime \prime} \times 181^{\prime \prime}\right)$, covering the same uv range and in which we subtracted the point sources detected at $25 \mathrm{~cm}$. In the $2 \mathrm{~m}$ map, the feature has a peak brightness of $28 \mathrm{mJy} /$ beam, while in the $85 \mathrm{~cm}$ map we can only give an upper limit, considering 3 times the noise of this map $\left(\sigma_{\mathrm{obs}}=1 \mathrm{mJy} /\right.$ beam $)$, i.e. $3 \mathrm{mJy} /$ beam. As a result, we obtain that the newly detected feature should have a spectrum steeper than -2.6 .

Because of the low resolution of the image, the NE relic seems to be embedded in the halo emission. The feature associated with it, already detected at $85 \mathrm{~cm}$ and tentatively at $25 \mathrm{~cm}$ (C2 in Fig. 4, bottom left panel), is now more prominent. However, we notice that, in this case, part of it could be due to the radio source located at RA $=17^{\mathrm{h}} 14^{\mathrm{m}} 04^{\mathrm{s}}$, Dec $=$ $+64^{\circ} 16^{\prime} 10^{\prime \prime}$, clearly detected at both $25 \mathrm{~cm}$ and $85 \mathrm{~cm}$.
The physical parameters of halo and NE relic at $2 \mathrm{~m}$ are listed in Table 5. We note that it is difficult to determine the real size and the borders of the halo and the NE relic, therefore the integrated flux densities reported in the table also reflect this uncertainty. The angular sizes of the two structures are assumed to be the same than at $85 \mathrm{~cm}$.

The NW relic is visible at $2 \mathrm{~m}$, while the SW one, which has a surface brightness lower than the NW relic at $85 \mathrm{~cm}$, is not detected $\left(S_{\mathrm{SW} \text { relic }}<6 \mathrm{mJy}\right)$. This is mainly due to the confusion limit in the central area of the map, where the noise level is $3 \mathrm{mJy}$.

Other extended features are detected to the E, NW and SW of the cluster center and at very large projected distance from it. The first one is located at RA $=17^{\mathrm{h}} 17^{\mathrm{m}} 25^{\mathrm{s}}$, Dec $=+64^{\circ} 05^{\prime} 15^{\prime \prime}$, the second one at RA $=17^{\mathrm{h}} 09^{\mathrm{m}} 29^{\mathrm{s}}, \mathrm{Dec}=+64^{\circ} 36^{\prime} 44^{\prime \prime}$ and the third one at RA $=17^{\mathrm{h}} 06^{\mathrm{m}} 10^{\mathrm{s}}$, Dec $=+63^{\circ} 18^{\prime} 42^{\prime \prime}$. Comparing the $2 \mathrm{~m}$ map with the full resolution $85 \mathrm{~cm}$ and $25 \mathrm{~cm}$ images, it 


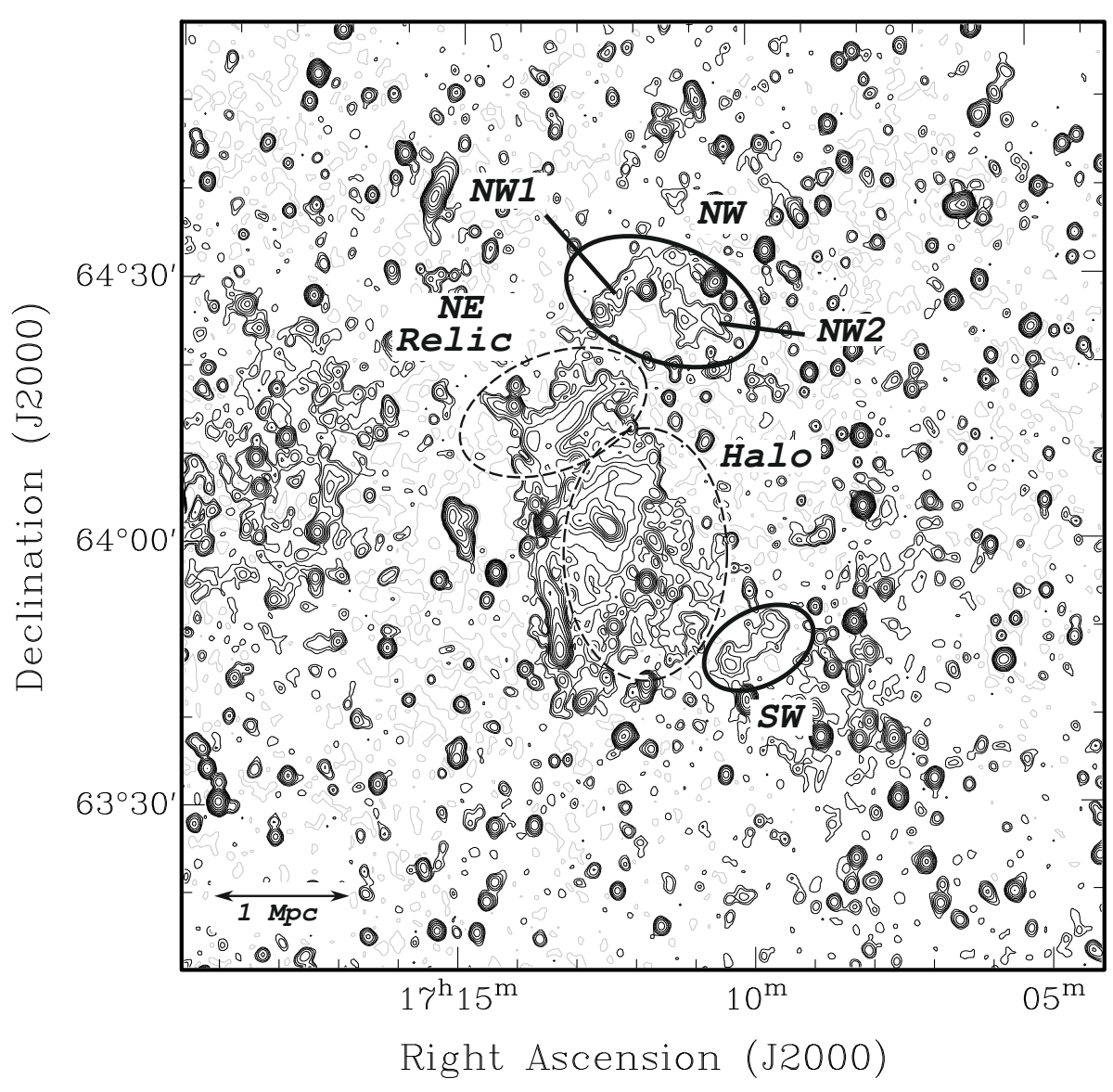

Fig. 6. Contour map of $\mathrm{A} 2255$ at $85 \mathrm{~cm}$. The resolution is $54^{\prime \prime} \times 64^{\prime \prime}$. The noise level at the edge of the fully imaged field is $\sim 0.1 \mathrm{mJy} /$ beam (see Fig. 2). The contours are -0.3 (grey), 0.3, 0.6, 1.2, 2.4, 4.8, 9.6, 20, 40, $80,160 \mathrm{mJy} / \mathrm{beam}$. The dotted ellipses indicate the structures already known through previous studies, as the halo and the NE relic, while the solid ellipses refer to the newly detected relics. We refer to the text for a discussion about the positive features at the east side of the cluster center. The map is not corrected for the primary beam.

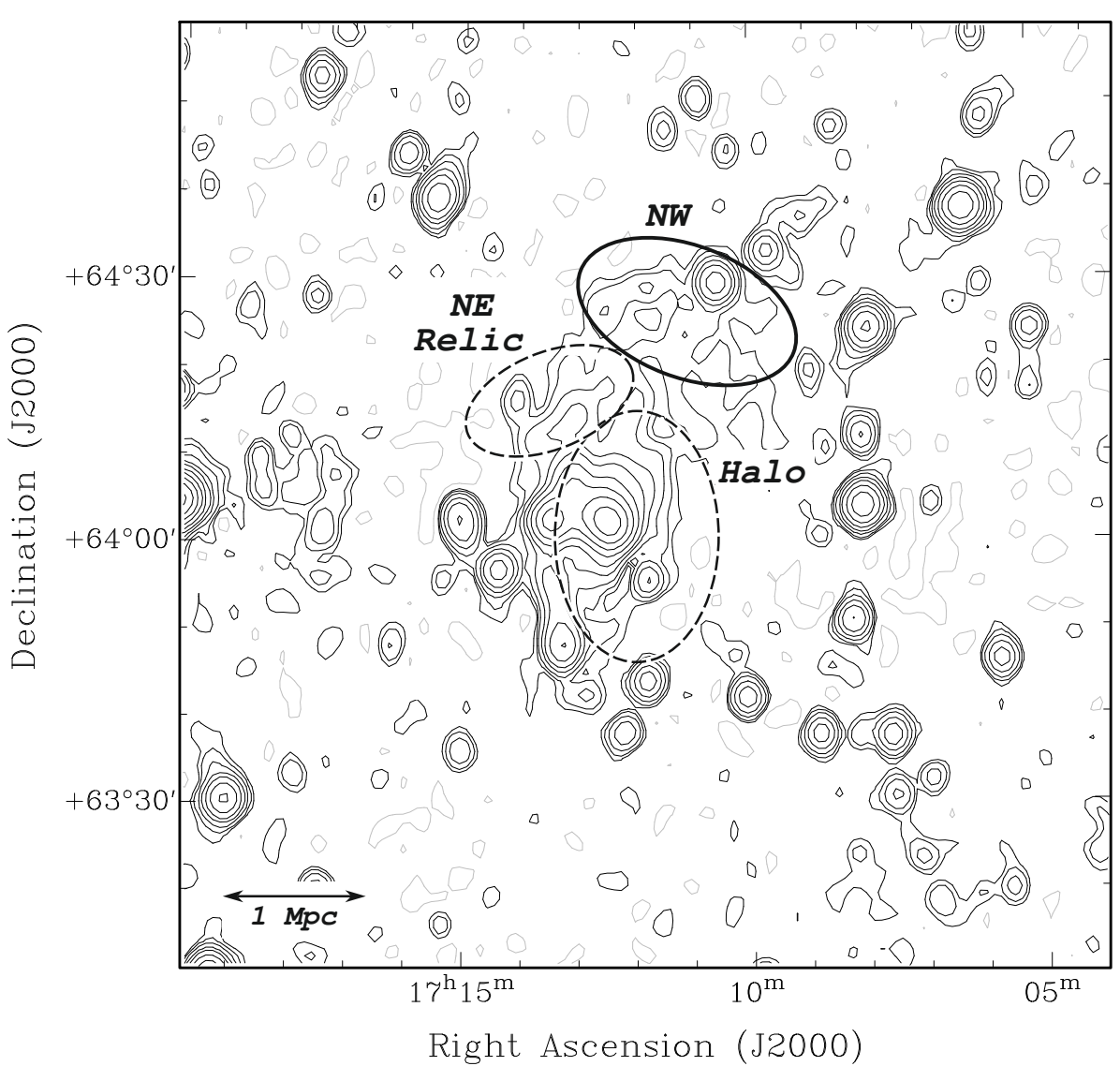

Fig. 7. Contour map of A2255 at $2 \mathrm{~m}$. The resolution is $163^{\prime \prime} \times 181^{\prime \prime}$. The noise level at the edge of the fully imaged field is $\sim 2 \mathrm{mJy} /$ beam (see Fig. 2). The contours are -0.007 (grey), $0.007,0.014,0.028,0.056,0.1,0.2,0.4,0.8$, $1.6,3.2 \mathrm{Jy} /$ beam. The radio halo is more extended towards NW than at $85 \mathrm{~cm}$ and is directly connected to the NW relic. The dotted lines indicate the structures already known through previous studies, as the halo and the NE relic, while the solid lines refer to the newly detected relics. The map is not corrected for the primary beam. 


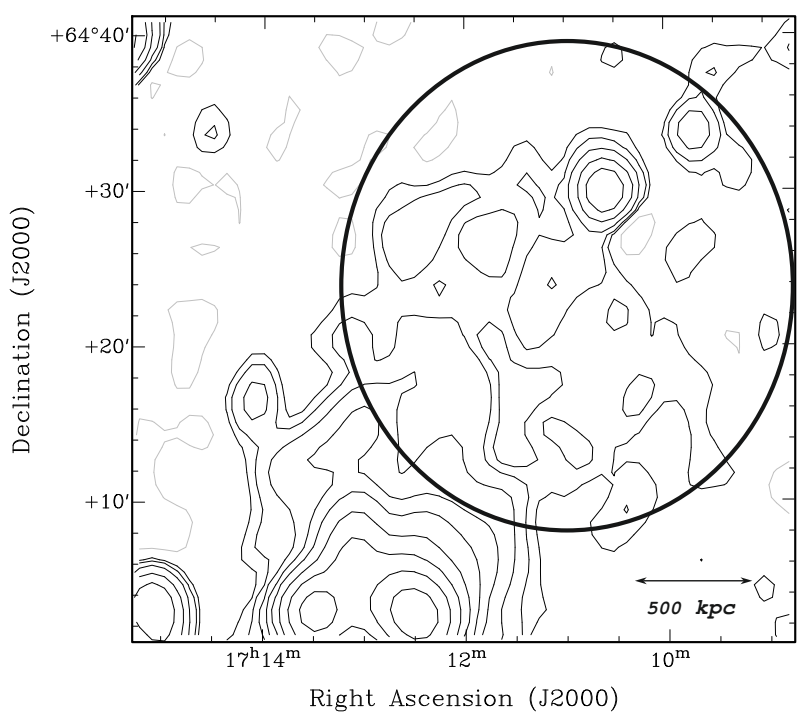

Fig. 8. Map at $2 \mathrm{~m}$ of the new extended emission region to the NW of the halo of A2255 after the subtraction of the model of point sources detected at $25 \mathrm{~cm}$ (see text). The result suggests that the new feature is genuine and not due to a collection of point sources. The resolution of the map is $163^{\prime \prime} \times 181^{\prime \prime}$. The contours are -0.007 (grey), $0.007,0.014$ $0.0280 .056,0.1,0.2,0.4,0.81 .63 .2 \mathrm{Jy} /$ beam. The map is not corrected for the primary beam. The circle represents the area within which the point sources have been subtracted.

Table 5. Parameters of the halo and the relics derived from the full resolution maps.

\begin{tabular}{cccc}
\hline \hline Source & Wavelength & $\begin{array}{c}\text { Angular size } \\
\left({ }^{\prime}\right)\end{array}$ & $\begin{array}{c}\text { Flux } \\
(\mathrm{mJy})\end{array}$ \\
\hline \multirow{3}{*}{ halo } & $25 \mathrm{~cm}$ & $7 \times 4$ & $44 \pm 1^{a}$ \\
& $85 \mathrm{~cm}$ & $17 \times 15$ & $496 \pm 7^{a}$ \\
& $2 \mathrm{~m}$ & $(17 \times 15)^{b}$ & $805 \pm 40^{a}$ \\
\hline \multirow{3}{*}{ NE relic } & $25 \mathrm{~cm}$ & $11 \times 3$ & $30 \pm 1$ \\
& $85 \mathrm{~cm}$ & $15 \times 4$ & $117 \pm 2$ \\
& $2 \mathrm{~m}$ & $(15 \times 4)^{b}$ & $287 \pm 17$ \\
\hline NW relic & $85 \mathrm{~cm}$ & $21 \times 1$ & $61 \pm 1$ \\
\hline SW relic & $85 \mathrm{~cm}$ & $13 \times 1$ & $17 \pm 1$ \\
\hline
\end{tabular}

${ }^{a}$ The fluxes have been determined avoiding the radio galaxies.

${ }^{b}$ Assumed to be the same as at $85 \mathrm{~cm}$. The low resolution of the $2 \mathrm{~m}$ map prevented a more precise determination of this parameter.

is evident that in this case we are dealing with unresolved point sources.

\section{Spectral index analysis of A2255}

After obtaining the final maps with the same resolution, uv min and gridding, we computed the spectral index images using the NRAO Astronomical Image Processing System (AIPS) package.

\subsection{Spectral index analysis between $25 \mathrm{~cm}$ and $85 \mathrm{~cm}$}

The spectral index image of A2255 between $25 \mathrm{~cm}$ and $85 \mathrm{~cm}$ is shown in Fig. 9.

We did not subtract the extended discrete radio sources, to avoid errors which might be introduced by the residuals of the subtraction. Therefore, we notice that the steep $\alpha$ values in the central area of the halo are probably associated with the tail of the "Original TRG". Moreover, the flat spectrum of the eastern regions of the halo, are heavily influenced by the presence of the nuclei of the Double and the Goldfish radio galaxies. For this reason, the only $\alpha$ values in the $\mathrm{N}$ and NW area of the halo can be considered as representative of the halo itself. Here, the spectrum shows a radial flattening from the center $(\alpha \sim-1.6 \pm 0.05)$ to the periphery ( $\alpha \sim-0.7 \pm 0.07$ ), where the three bright radio filaments are detected at high-frequency (see Fig. 4). At this location, fresh (re-)acceleration of relativistic particles is expected to take place, producing a flattening of the spectrum.

The NE relic shows $\alpha$ values which range from $-1.46 \pm 0.08$ to $-0.4 \pm 0.09$. There is a gradient of the spectral index along the main axis: $\alpha$ flattens from south-east towards north-west (see right panel of Fig. 11). Moreover, $\alpha$ shows a trend also along the minor axis, being steeper in the regions close to the cluster center and flattening towards the periphery (see left panel of Fig. 11).

As discussed in Sect. 3.1, the brighter filament of the NW relic is detected at $25 \mathrm{~cm}$ as a very low brightness feature ( $1 \sigma /$ beam). Because of the $3 \sigma$ criterium we used to produce the spectral index map between $25 \mathrm{~cm}$ and $85 \mathrm{~cm}$, this feature is not visible in the low resolution map.

\subsection{Spectral index analysis between $85 \mathrm{~cm}$ and $2 \mathrm{~m}$}

Figure 10 shows the spectral index image of A2255, calculated between $2 \mathrm{~m}$ and $85 \mathrm{~cm}$. The spectral index image has been obtained clipping the pixels where the brightness was below $3 \sigma$ level at both wavelengths.

The spectral index within the halo shows a more patchy structure than between $25 \mathrm{~cm}$ and $85 \mathrm{~cm}$. The $\alpha$ values are flatter in the regions corresponding to the nuclei of the central radio galaxies $(\alpha=-0.9 \pm 0.01)$ and steepen in between ( $\alpha=-1.6 \pm 0.01)$. In the NW regions of the halo, where there is no contamination from radio galaxies, $\alpha$ shows a discontinuous behavior, ranging between $-2.8 \pm 0.1$ and $-1.6 \pm 0.07$. As for the spectral index image between $25 \mathrm{~cm}$ and $85 \mathrm{~cm}$, the flatter values are confined to a semi circular area that approximately describes the location of the 3 bright filaments at high-frequency.

The NE relic shows $\alpha$ values in the range of $-0.9 \pm 0.1$ to $-1.2 \pm 0.1$, with the steeper values confined in its NW areas. We notice, however, that because of the criteria used to make the spectral index map, combined with the high noise level in the central part of the $2 \mathrm{~m}$ map, $\alpha$ has been determined for the central area of the NE relic only. Moreover, given the high rms values associated with the spectral index in its SE region, no clear radial trend neither along the main axis nor perpendicular to it can be determined.

The NW relic, detected at $2 \mathrm{~m}$ in the high sensitivity map, is no longer entirely visible and only a small part of the filament pointing towards the cluster center is still detected. Its spectral index ranges between $-1.2 \pm 0.2$ and $-0.5 \pm 0.1$, and steepens towards the cluster center. Moreover, there is a feature at location $\mathrm{RA}=17^{\mathrm{h}} 11^{\mathrm{m}} 00^{\mathrm{s}}$, Dec $=+64^{\circ} 24^{\prime} 49^{\prime \prime}$, which is likely associated with the second filament perpendicular to the previous one. Here, $\alpha$ steepens towards the cluster center and ranges between $-1.8 \pm$ 0.2 and $-0.6 \pm 0.3$.

We determined the integrated synchrotron spectrum of the region of the radio halo without the contamination of radio galaxies and of the NE relic in the wavelength range $2 \mathrm{~m}-25 \mathrm{~cm}$ (Fig. 12). The fluxes of the analyzed features are reported in Table 6. The spectrum of the radio halo has a constant slope between the 3 wavelengths $\left(\alpha_{85 \mathrm{~cm}}^{2 \mathrm{~m}}=-1.30 \pm 0.05\right.$ and $\alpha_{85 \mathrm{~cm}}^{25 \mathrm{~cm}}=-1.30 \pm 0.1$ ), while the one of the NE relic is flatter at 


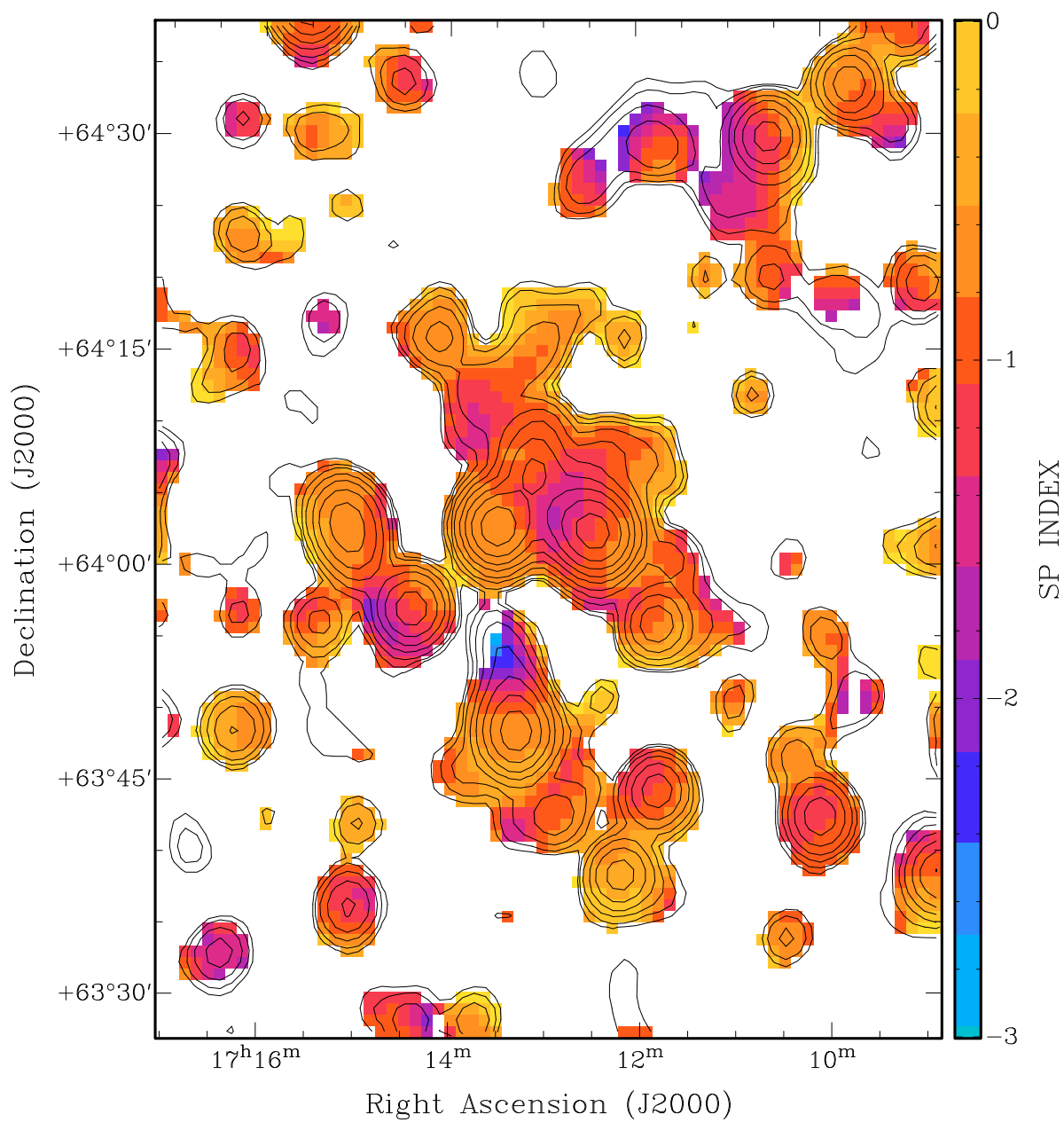

Fig. 9. Spectral index map of A2255 between $25 \mathrm{~cm}$ and $85 \mathrm{~cm}$, with a resolution of $163^{\prime \prime} \times$ $181^{\prime \prime}$. Pixels whose brightness was below $3 \sigma$ at $25 \mathrm{~cm}$ or $85 \mathrm{~cm}$ have been blanked. The cut is driven by the $85 \mathrm{~cm}$ image in most of the points. Contour levels are the ones of the radio map at $85 \mathrm{~cm}$ at low resolution: $0.0015(3 \sigma), 0.003$, $0.006,0.012,0.024,0.048,0.096,0.18,0.36$, $0.72,1.4 \mathrm{Jy} /$ beam. low-frequency $\left(\alpha_{85 \mathrm{~cm}}^{2 \mathrm{~m}}=-0.5 \pm 0.2\right)$ and steepens at highfrequency $\left(\alpha_{85 \mathrm{~cm}}^{25 \mathrm{~cm}}=-0.81 \pm 0.1\right)$.

\subsection{Equipartition magnetic field}

The total energy content of a synchrotron source $\left(U_{\text {tot }}\right)$ is given by the contribution of the energy of the relativistic particles and the energy of the magnetic field. $U_{\text {tot }}$ shows a minimum when these 2 contributions are approximately equal. For this reason the minimum energy is known also as equipartition value. If we assume that a radio source is in a condition of minimum energy, it is possible to estimate the magnetic field strength (Pacholczyk 1970). The equipartition magnetic field $\left(B_{\text {eq }}\right)$ is expressed by

$B_{\text {eq }}=\left(\frac{24 \pi}{7} u_{\min }\right)^{1 / 2}$,

where the total minimum energy density $\left(u_{\min }\right)$ of the source depends on observational quantities, as the source brightness $\left(I_{0}\right)$, its redshift $(z)$, and the observing frequency $\left(v_{0}\right)$, and on unknown parameters, like the ratio between the energy in relativistic protons and electrons $(k)$ and the filling factor $(\Phi)$, which represents the fraction of the source volume that is filled with particles and magnetic fields:

$$
\begin{aligned}
u_{\min }\left[\frac{\mathrm{erg}}{\mathrm{cm}^{3}}\right]= & \xi\left(\alpha, v_{1}, v_{2}\right)(1+k)^{4 / 7}\left(v_{0[\mathrm{MHz}]}\right)^{-4 \alpha / 7}(1+z)^{(12-4 \alpha) / 7} \\
& \times\left(I_{0\left[\frac{\mathrm{mJy}}{\operatorname{arcscc}^{2}}\right]}\right)^{4 / 7}\left(d_{[\mathrm{kpc}]}\right)^{-4 / 7} \Phi^{-4 / 7} .
\end{aligned}
$$

Here, $\xi\left(\alpha, v_{1}, v_{2}\right)$ is a constant which is tabulated in Govoni \& Feretti (2004) and $d$ is the source depth. The latter formula is mainly used for significantly extended structures. In the case of compact features, Eq. (7) is better expressed by

$$
\begin{aligned}
u_{\min }\left[\frac{\mathrm{erg}}{\mathrm{cm}^{3}}\right]= & \xi\left(\alpha, v_{1}, v_{2}\right)(1+k)^{4 / 7}\left(v_{0[\mathrm{MHz}]}\right)^{-4 \alpha / 7}(1+z)^{(12-4 \alpha) / 7} \\
& \times\left(I_{0[\mathrm{mJy}]}\right)^{4 / 7}\left(V_{\left[\mathrm{kpc}^{3}\right]}\right)^{-4 / 7} \Phi^{-4 / 7}
\end{aligned}
$$

where $V$ is the source volume.

Table 6 lists the equipartition magnetic field for the region of the halo free of radio galaxies and for the NE relic. It was computed using the fluxes at $85 \mathrm{~cm}$. Furthermore, we assumed equal energy in relativistic protons and electrons $(k=1)$ and a filling factor of unity ( $\Phi=1$, the volume is homogeneously filled by the relativistic plasma). The synchrotron luminosity is calculated in the frequency range $10 \mathrm{MHz}-100 \mathrm{GHz}$. Halo and relic are shaped as cylinders with a size (length $\times$ depth, derived from the map at $25 \mathrm{~cm}$ ) of $630 \times 360 \mathrm{kpc}$ and $990 \times 270 \mathrm{kpc}$, respectively. A spectral index of $\alpha=-1.3$ for the halo and $\alpha=$ -0.7 for the NE relic was adopted.

Govoni et al. (2006) studied the intra cluster magnetic field power spectrum of A2255 through the analysis of the RM distributions of three cluster radiogalaxies. They found that to reproduce the behavior of the RM for the radio galaxies, the power spectrum of the magnetic field of the cluster should steep from the center to the periphery, with an average magnetic field strength for the radio halo calculated over $1 \mathrm{Mpc}^{3}$ of about $1.2 \mu \mathrm{G}$. This value is a factor of 2 higher than the equipartition magnetic field obtained by us. The discrepancy 


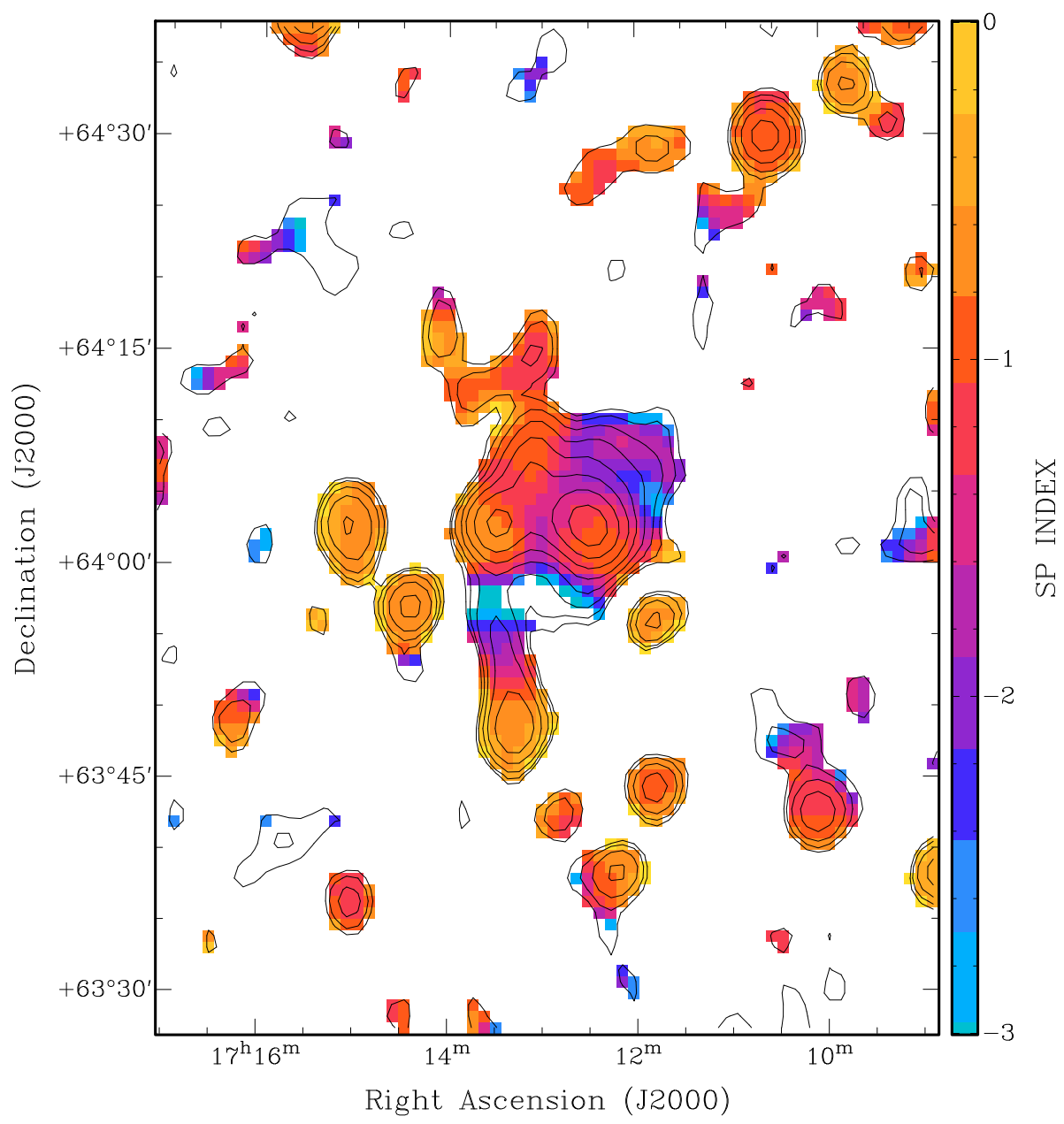

Fig. 10. Spectral index map of A2255 between $85 \mathrm{~cm}$ and $2 \mathrm{~m}$, with a resolution of $163^{\prime \prime} \times$ $181^{\prime \prime}$. Pixels whose brightness was below $3 \sigma$ at $85 \mathrm{~cm}$ or $2 \mathrm{~m}$ have been blanked. The cut is driven by the $2 \mathrm{~m}$ image in most of the points. Contour levels are the ones of the radio map at $2 \mathrm{~m}: 0.015(3 \sigma), 0.030,0.06,0.12,0.24,0.48$, $0.96,1.8,3.6 \mathrm{Jy} / \mathrm{beam}$.

is mainly due to the fact that, in the approach followed by Govoni et al. (2006), also low energy relativistic particles are taken into account when computing the strength of the magnetic field, while in the standard approach used in the computation of the equipartition parameters one considers a cut frequency window between $v_{1}$ and $v_{2}$. Brunetti et al. (1997) demonstrated that it is more appropriate to calculate the radio source energy by integrating the synchrotron luminosity over a range of electron energies. This method has the advantage that electrons of very low energy are also taken into account and it avoids the problem that electron energies corresponding to frequencies $v_{1}$ and $v_{2}$ depend on the magnetic field value. Representing the electron energy with its Lorentz factor $\gamma$, and assuming that $\gamma_{\min } \ll \gamma_{\max }$, the new expression of the equipartition magnetic field is

$B_{\mathrm{eq}}^{\prime} \sim 1.1 \gamma_{\min }^{\frac{1+2 \alpha}{3-\alpha}} B_{\mathrm{eq}}^{\frac{7}{2(3-\alpha)}}$,

where $B_{\text {eq }}^{\prime}$ and $B_{\text {eq }}$ are expressed in Gauss. Using Eq. (9), we find that the equipartition magnetic field computed by us and the average magnetic field of the halo calculated by Govoni et al. (2006) are in agreement if we assume a cut in the energy distribution of the relativistic particles at $\gamma_{\min }=310$. By using the same $\gamma_{\min }$ as for the radio halo, the new equipartition magnetic field for the NE relic is $\sim 0.6 \mu \mathrm{G}$.

\section{The head-tail Beaver radio galaxy}

Abell 2255 is one of the richest clusters of the Abell catalog in terms of radio galaxies. In our maps we can detect 7 radio galaxies: four are located in the central region of the cluster, and three lie at large projected distance from it. The physical properties of these radio galaxies have already been studied by several authors (Harris et al. 1980; Feretti et al. 1997; Miller \& Owen 2003), but so far spectral index images were not presented in the literature. During the analysis of our multi-frequency observations, we studied the physical properties of the Beaver radio galaxy because its long tail gave us the possibility to test ageing models of the radiating electrons.

\subsection{Spectral index analysis}

One of the most interesting properties of the Beaver is that it doubles the length of its tail to almost $1 \mathrm{Mpc}$ between $25 \mathrm{~cm}$ and $85 \mathrm{~cm}$ (see Fig. 13). This suggests very steep $\alpha$ values for the ending part of the tail, which is an indication of severe energy losses suffered by the relativistic particles.

In Figs. 14 and 15, we show the spectral index maps of the Beaver, between $25 \mathrm{~cm}$ and $85 \mathrm{~cm}$, and between $85 \mathrm{~cm}$ and $2 \mathrm{~m}$. The spectral index distribution clearly confirms that we are dealing with a tailed radio galaxy, since $\alpha$ severely steepens going from the head of the source towards the end of the tail. Between $25 \mathrm{~cm}$ and $85 \mathrm{~cm}$, the spectral index of the Beaver could be computed for the head and the initial part of the tail. As we expect, $\alpha$ is flatter in the regions closer to the nucleus ( $\alpha=-0.5 \pm 0.08$ ), where more energetic electrons are continuously injected by the central AGN, and then it steepens reaching values of $\alpha=-2.2 \pm 0.2$. We would have expected a flatter spectral index for the head of the radio galaxy, but the low resolution of the maps makes the final spectral index of the nucleus contaminated by the surrounding steeper regions. The spectral index 

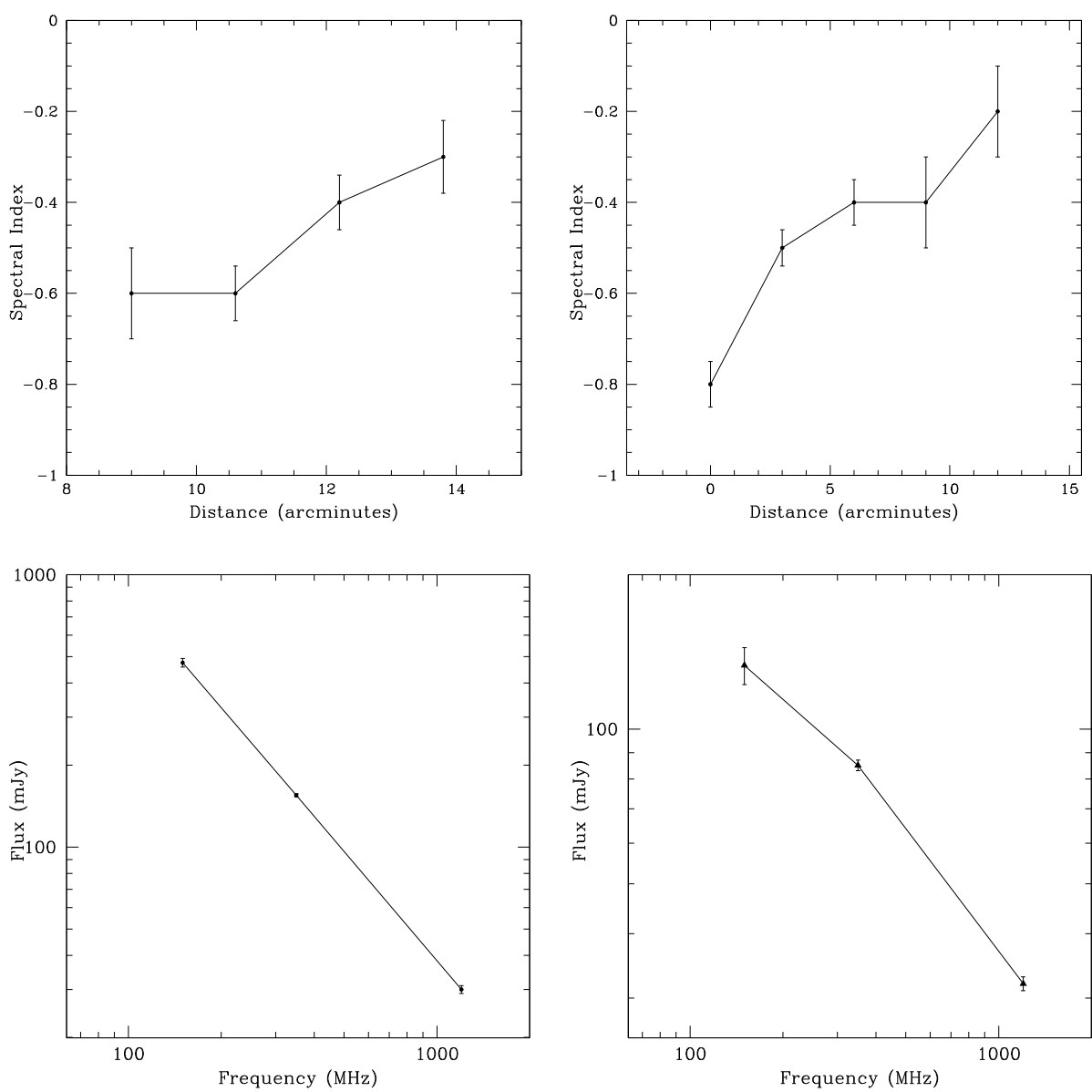

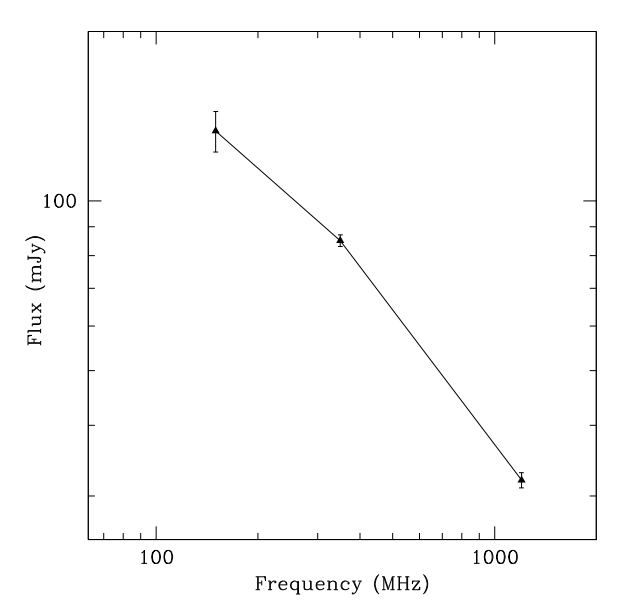

Fig. 11. Spectral index profile of the NE relic between $25 \mathrm{~cm}$ and $85 \mathrm{~cm}$ along the minor axis (left panel) and the main axis (right panel). For the former the distance is from the cluster center, for the latter the distance is from position $\mathrm{RA}=17^{\mathrm{h}} 13^{\mathrm{m}} 51^{\mathrm{s}}$, Dec $=+64^{\circ} 12^{\prime} 00^{\prime \prime}$, which is the SE edge of the relic.

Table 6. Parameters of the NW region of the halo and of the NE relic derived from the images produced with the same uv coverage and the same resolution.

\begin{tabular}{|c|c|c|c|c|c|c|c|c|c|c|}
\hline Region Flux & $\underset{\substack{\mathrm{h} \mathrm{m} \mathrm{s} \\
\mathrm{RA}_{\mathrm{J}}}}{ }$ & $\operatorname{Dec}_{\substack{J 2000 \\
0, \prime \prime}}$ & $\begin{array}{l}\text { Size } \\
(\mathrm{kpc})\end{array}$ & $\begin{array}{c}S_{\text {tot }}^{\lambda=2 \mathrm{~m}} \\
\mathrm{mJy}\end{array}$ & $\begin{array}{c}S_{\text {tot }}^{\lambda=85 \mathrm{~cm}} \\
\mathrm{mJy}\end{array}$ & $\begin{array}{c}S_{\text {tot }}^{\lambda=25 \mathrm{~cm}} \\
\mathrm{mJy}\end{array}$ & $\alpha_{85 \mathrm{~cm}}^{2 \mathrm{~m}}$ & $\alpha_{25 \mathrm{~cm}}^{85 \mathrm{~cm}}$ & $\begin{array}{c}u_{\min } \\
\left(\mathrm{erg} / \mathrm{cm}^{3}\right)\end{array}$ & $\begin{array}{r}B_{\mathrm{eq}} \\
(\mu \mathrm{G}) \\
\end{array}$ \\
\hline Radio Halo $^{a}$ & 171300 & 640759 & $630 \times 360$ & $475 \pm 17$ & $155 \pm 2$ & $30 \pm 1$ & $-1.3 \pm 0.05$ & $-1.3 \pm 0.1$ & $3.3 \times 10^{-14}$ & 0.6 \\
\hline NE Radio Relic & 171322 & 641336 & $990 \times 270$ & $133 \pm 11$ & $85 \pm 1$ & $32 \pm 1$ & $-0.5 \pm 0.1$ & $-0.8 \pm 0.1$ & $1.4 \times 10^{-15}$ & 0.4 \\
\hline
\end{tabular}

${ }^{a}$ For the halo the parameters have been determined avoiding the radio galaxies.

between $85 \mathrm{~cm}$ and $2 \mathrm{~m}$ shows basically the same trend, being flatter in the nucleus region $(\alpha \sim-0.6 \pm 0.03)$ and steepening towards the cluster radio halo $(\alpha \sim-3.3 \pm 0.2)$.

The integrated spectra of the head and the tail of the Beaver are shown in Fig. 16. The trend of the spectral index and of the primary beam corrected brightness between $2 \mathrm{~m}$ and $85 \mathrm{~cm}$ along the tail is shown in Fig. 17. We computed $\alpha$ at 3 different positions, starting from $4^{\prime}$ from the nucleus of the radio galaxy and going towards the end of the tail, averaging the $\alpha$ values within beam size boxes.

\subsection{Physical parameters and spectral ageing in the tail of the Beaver}

The electrons in the tail of the radio galaxies are not thought to be subjected to large bulk motions. Their number in a particular region of the tail remains constant after the galaxy has passed. Therefore, their position can be considered a measure of the age of the electrons.

Given a homogeneous and isotropic population of electrons with a power law energy distribution $\left(N(E) \mathrm{d} E=N_{0} E^{-\gamma} \mathrm{d} E\right)$, the synchrotron spectrum for regions optically thin to their own radiation varies with frequency as

$J(v) \propto N_{0}(B \sin \theta)^{1-\alpha} v^{\alpha}$,

where $\theta$ is the pitch angle between the electron velocity and the magnetic field direction. The spectral index $\alpha$ is related to the index of the electron energy distribution: $\alpha=(1-\gamma) / 2$.

The electron energy decreases with time and the resulting synchrotron spectrum undergoes to a modification. One will observe a critical frequency $v^{*}$, such that for $v<v^{*}$ the spectrum is unchanged, whereas for $v>v^{*}$ the spectrum steepens.

There are several mechanisms that can make the electrons loose their energy: synchrotron radiation, inverse Compton scattering, adiabatic expansion, Bremstrahlung and ionization losses (Kardashev 1962; Pacholczyk 1970). However, for confined tails the first two mechanisms play the most important role.

There are mainly two models that describe the steepening of the radio spectrum above $v^{*}$ :

- the Kardashev-Pacholczyk model (KP model, Kardashev 1962; Pacholczyk 1970), in which the electrons maintain the 


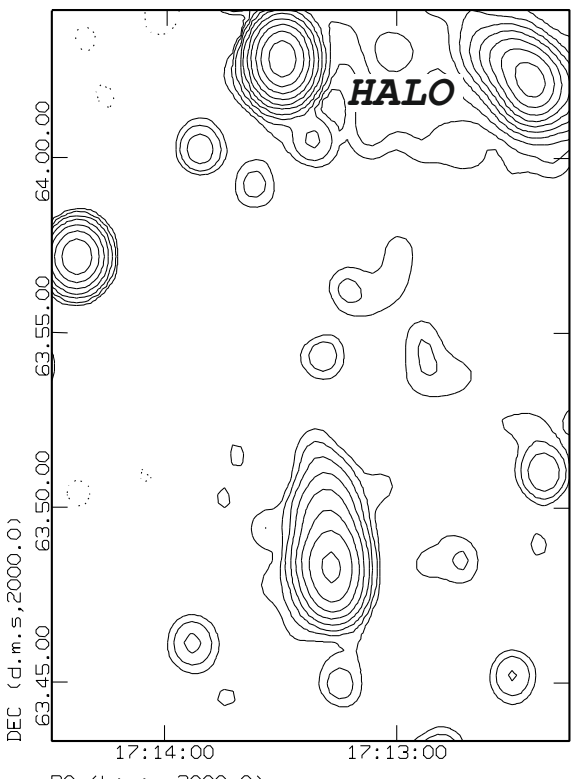

$R A(h: m: 5,2000.0)$

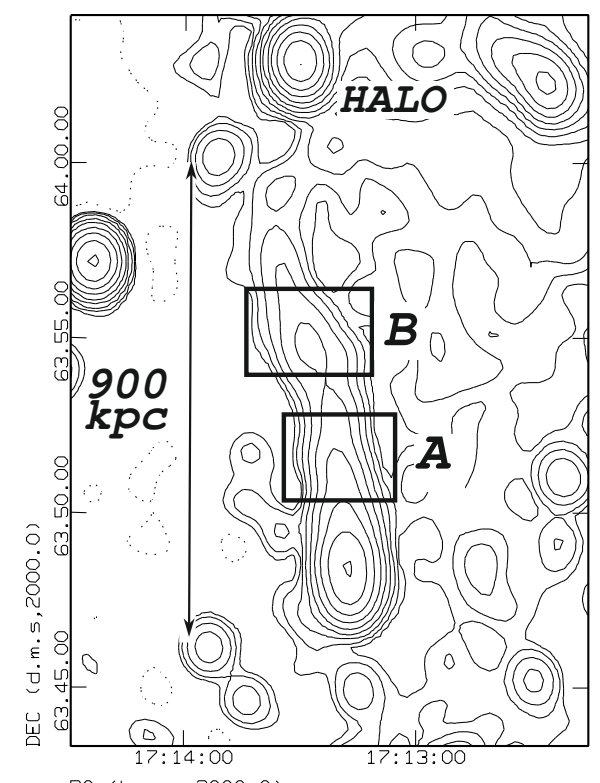

RA (h:m:s,2000.0)
Fig. 13. Comparison between the morphologies of the Beaver radio galaxy at $25 \mathrm{~cm}$ (left panel) and $85 \mathrm{~cm}$ (right panel). The $25 \mathrm{~cm}$ map has been convolved with the $85 \mathrm{~cm}$ beam $\left(54^{\prime \prime} \times 64^{\prime \prime}\right)$. Boxes A and B define two regions along the tail where we did our analysis (see Sects. 5.3-5.2). At $25 \mathrm{~cm}$, the contours are $-0.25,0.25,0.50,1,2,4,8,16,32,64$, $128 \mathrm{mJy} / \mathrm{beam}$; at $85 \mathrm{~cm},-0.5,0.5,1,2,4,8$, $16,32,64,128,256,512 \mathrm{mJy} / \mathrm{beam}$. The images are not corrected for the primary beam.

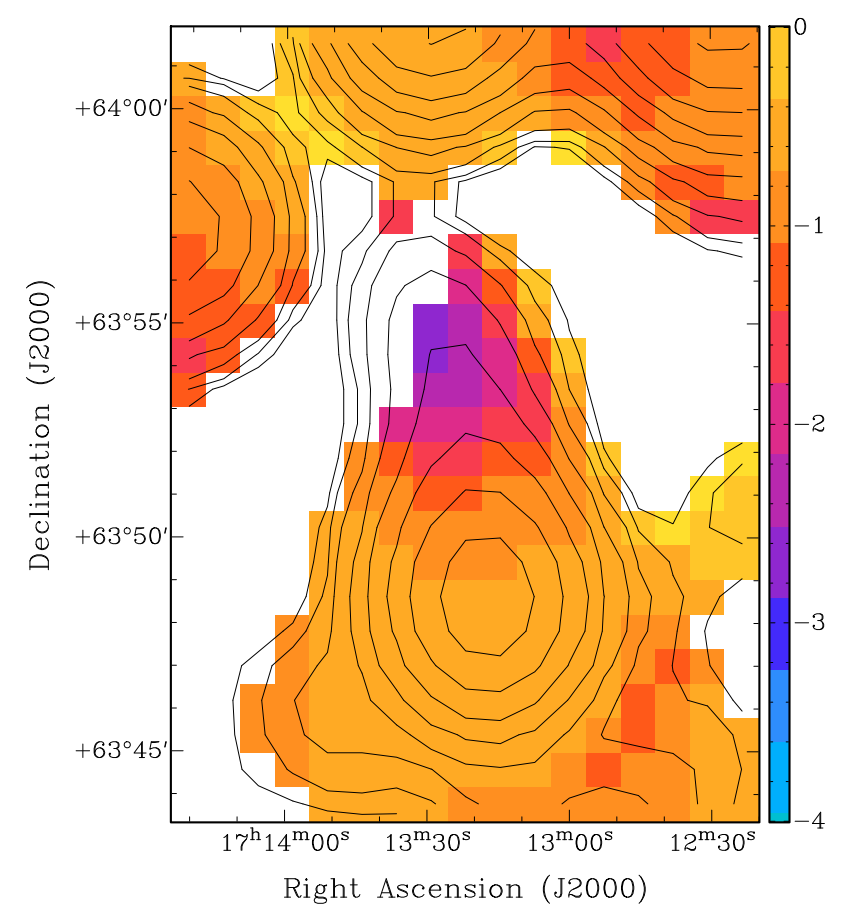

Fig. 14. Spectral index map of the Beaver radio galaxy between $25 \mathrm{~cm}$ and $85 \mathrm{~cm}$, with a resolution of $163^{\prime \prime} \times 181^{\prime \prime}$. Pixels whose brightness was below $3 \sigma$ at $25 \mathrm{~cm}$ or $85 \mathrm{~cm}$ have been blanked. The cut is driven by the $85 \mathrm{~cm}$ image in most of the points. Contour levels are the ones of the radio map at $85 \mathrm{~cm}$ at low resolution: $0.0015(3 \sigma), 0.003,0.006$, $0.012,0.024,0.048,0.096,0.18,0.36,0.72,1.4 \mathrm{Jy} /$ beam.

same pitch angle with respect to the B lines. The particles will have different energy losses depending on the value of this parameter;

- the Jaffe-Perola model (JP model, Jaffe E Perola 1973), where one considers an electrons population with an isotropic distributions of pitch angles. This results in a sharp energy cutoff in the energy electron distribution, with a synchrotron spectrum showing an exponential drop at highfrequency.

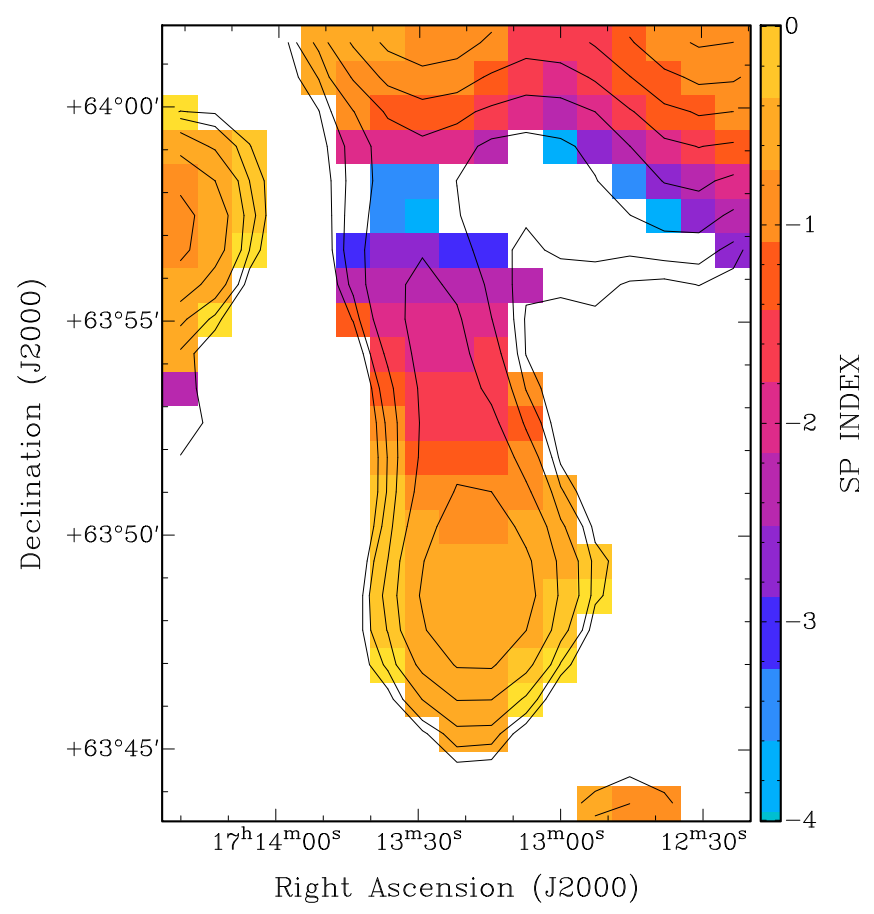

Fig. 15. Spectral index map of the Beaver radio galaxy between $85 \mathrm{~cm}$ and $2 \mathrm{~m}$, with a resolution of $163^{\prime \prime} \times 181^{\prime \prime}$. Pixels whose brightness was below $3 \sigma$ at $85 \mathrm{~cm}$ or $2 \mathrm{~m}$ have been blanked. The cut is driven by the $2 \mathrm{~m}$ image in most of the points. Contour levels are the ones of the radio map at $2 \mathrm{~m}$ : $0.015(3 \sigma), 0.030,0.06,0.12,0.24,0.48,0.96,1.8$, $3.6 \mathrm{Jy} /$ beam.

Since the electrons within a radio source are likely to have various distributions of the pitch angle, the JP model better describes this physical situation. Ignoring adiabatic losses and assuming a constant magnetic field with time, for the JP model the cutoff energy is:

$E_{\mathrm{c}}[\mathrm{GeV}]=1.3 \times 10^{10} \frac{1}{\left(B_{\mathrm{eq}}^{2}+B_{\mathrm{IC}}^{2}\right) t}$ 

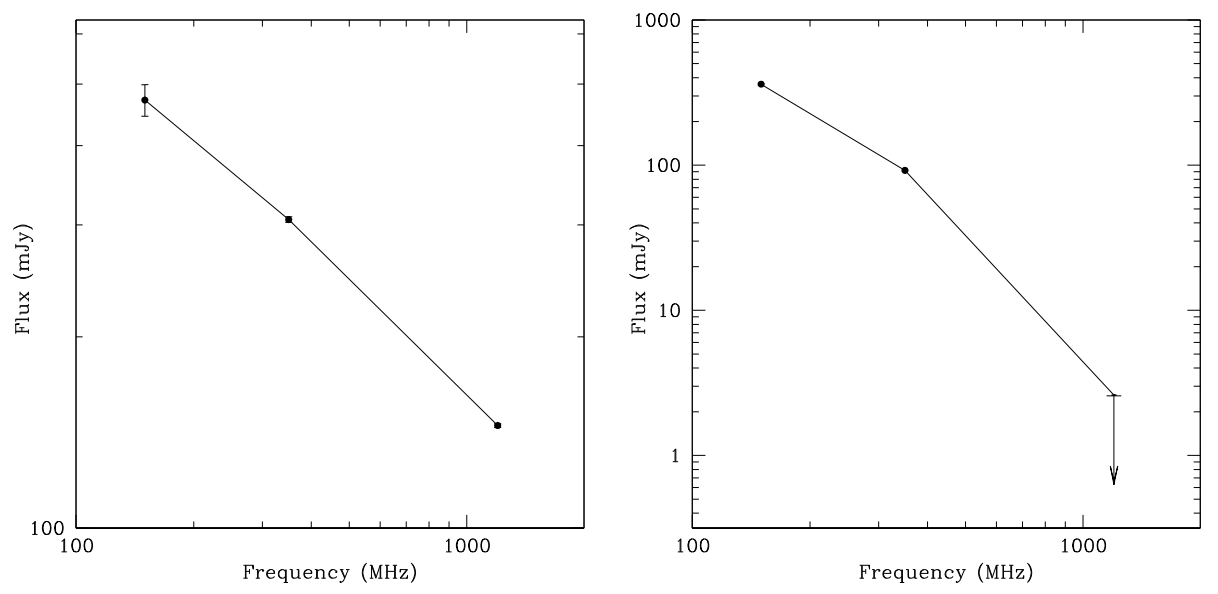

Fig. 16. Radio spectra of the head (left panel) and the tail (right panel) of the Beaver radio galaxy in the frequency range
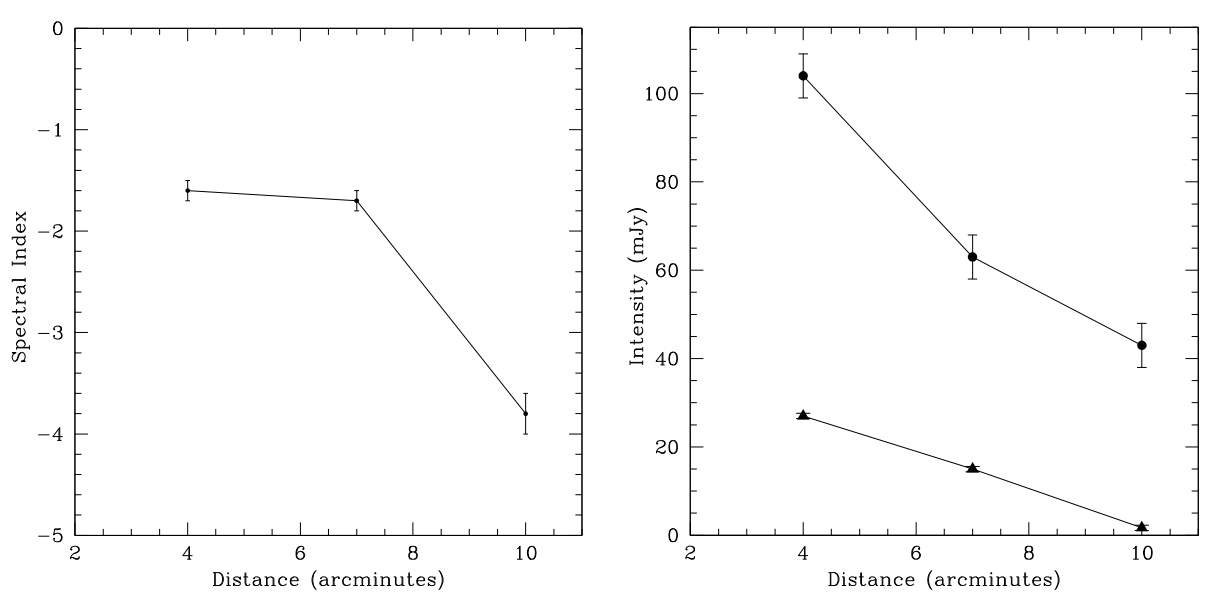
$2 \mathrm{~m}-25 \mathrm{~cm}$

Fig. 17. Right panel: point to point spectral index between $85 \mathrm{~cm}$ and $2 \mathrm{~m}$. The $\alpha$ values are plotted versus the distance from the nucleus of the Beaver, in arcminutes. Right panel: variation of the primary beam corrected brightness at $2 \mathrm{~m}$ (top line) and $85 \mathrm{~cm}$ (bottom line).

where $B_{\text {IC }}$ represents the equivalent magnetic field strength of the microwave background and is expressed in $\mu \mathrm{G}$, as well as $B_{\text {eq. }}$.

The break frequency is

$$
v^{*}(\mathrm{MHz})=16.08 B_{\mathrm{eq}} E_{\mathrm{c}}^{2} \text {. }
$$

We have fitted the integrated fluxes of the Beaver for two regions along the tail (labeled A and B in Fig. 13). They have been selected far from any possible contaminant source, as nucleus and extended emitting areas of the cluster radio halo. The tail of the Beaver was not detected at $25 \mathrm{~cm}$ in region $\mathrm{B}$, thus the flux we used in this case is an upper limit. To produce the fit, the break frequency and the spectral index in the part of the spectrum not affected by any evolution $\left(\alpha_{\text {inj }}\right)$ are left as free parameters. The result is shown in Fig. 18. The very low $\chi^{2}$ value may be due to the number of free parameters used in the fit. For region A, the best fit is obtained for $\alpha_{\mathrm{inj}}=-0.5$ and a break frequency $v^{*}=353.6 \mathrm{MHz}$. For region $\mathrm{B}$, we have assumed the same injection spectral index than region $\mathrm{A}$, obtaining $v^{*}=94.52 \mathrm{MHz}$.

From the shape of its synchrotron spectrum and under the assumption of equipartition, it is possible to estimate the radiative age of the radio source. For the JP model, this is given by

$t_{\mathrm{r}}=\frac{1.6 \times 10^{9}}{B^{2}+B_{\mathrm{IC}}^{2}} \sqrt{\frac{B}{(1+z) v^{*}}} \quad[\mathrm{yr}]$,

where $B$ is in $\mu \mathrm{G}$ and $v$ in $\mathrm{GHz}$. Assuming a CMB temperature of $2.725 \mathrm{~K}, B_{\mathrm{IC}}=3.238(1+z)^{2} \mu \mathrm{G}$. For regions $\mathrm{A}$ and $\mathrm{B}$, we obtain the radiative ages $t_{r_{\mathrm{a}}} \sim 1.9 \times 10^{8} \mathrm{yr}$ and $t_{r_{\mathrm{b}}} \sim 2.2 \times 10^{8} \mathrm{yr}$, respectively. For this calculation, we assumed $B$ to be the equipartition magnetic field. $B_{\text {eq }}$ is reported in Table 7 , together with the other physical parameters for regions A and B, and the head of the Beaver. The equipartition parameters were computed using the fluxes at $85 \mathrm{~cm}$ (Col. 4). We shaped the head of the source with a spherical geometry with a diameter of $15 \mathrm{kpc}$, while for the tail we assumed a double cylinder geometry, with each cylinder having a depth of $25 \mathrm{kpc}$ and a length of $360 \mathrm{kpc}$. The sizes of the structures were derived from the high resolution $\left(2^{\prime \prime} \times 2^{\prime \prime}\right)$ image of the Beaver in Fig. 1 in Govoni et al. (2006).The depth and the width are assumed to be the same for each feature. To avoid any overestimation of the physical parameters, we adopted a spectral index which is the average of $\alpha_{85 \mathrm{~cm}}^{2 \mathrm{~m}}$ and $\alpha=-0.7$. For position $\mathrm{B}$, we assumed a spectral index $\alpha=-0.5$, which is the injection spectral index as for position $\mathrm{A}$.

An estimation of the kinematic age of the Beaver can be obtained assuming that the host galaxy traveled from position B till its current location ( $D=900 \mathrm{kpc}$ ) with the constant velocity of $\sim 1000 \mathrm{~km} \mathrm{~s}^{-1}$ (the velocity dispersion of A2255 is $\left.\sigma_{\mathrm{v}_{\mathrm{A} 2255}}=1266 \mathrm{~km} \mathrm{~s}^{-1}\right)$. This implies $t_{\mathrm{kin}} \sim 6 \times 10^{8} \mathrm{yr}$.

\subsection{Radio source confinement}

An important connection between the ICM and the radio galaxies is provided by the thermal pressure of the gas. From the radio data it is possible to derive the pressure within the radio source, under the assumption that the radio galaxy is in equipartition. From X-ray data, one can estimate the parameters of the intergalactic medium surrounding the radio source. By comparing the internal pressure of the radio emitting plasma with the thermal pressure of the ambient gas, we can get information about the equilibrium between thermal and nonthermal plasma. 
Table 7. Fluxes and equipartition parameters of the Beaver radio galaxy.

\begin{tabular}{cccccccccc}
\hline \hline Region & $\begin{array}{c}\text { Size } \\
(\mathrm{kpc})\end{array}$ & $\begin{array}{c}S_{2 \mathrm{~m}} \\
(\mathrm{mJy})\end{array}$ & $\begin{array}{c}S_{85 \mathrm{~cm}} \\
(\mathrm{mJy})\end{array}$ & $\begin{array}{c}S_{25 \mathrm{~cm}} \\
(\mathrm{mJy})\end{array}$ & $\alpha_{85 \mathrm{~cm}}^{2 \mathrm{~m}}$ & $\alpha_{25 \mathrm{~cm}}^{85 \mathrm{~cm}}$ & $\begin{array}{c}u_{\min }{ }^{a}{ }^{3} \\
\left(\mathrm{erg} / \mathrm{cm}^{-3}\right)\end{array}$ & $\begin{array}{c}B_{\mathrm{eq}}{ }^{b} \\
(\mu \mathrm{G})\end{array}$ & $\begin{array}{c}P_{\mathrm{eq}}{ }^{c} \\
\left(\mathrm{dyne} \mathrm{cm}^{-2}\right)\end{array}$ \\
\hline Head & $15 \times 15$ & $472 \pm 13$ & $306 \pm 4$ & $145 \pm 2$ & $-0.5 \pm 0.1$ & $-0.6 \pm 0.1$ & $8.1 \times 10^{-11}$ & 30 & $2.7 \times 10^{-11}$ \\
$\mathrm{~A}$ & $2^{d} \times(25 \times 360)$ & $113 \pm 6$ & $35 \pm 1$ & $1.2 \pm 0.2$ & $-1.4 \pm 0.1$ & $-2.7 \pm 1.1$ & $1.9 \times 10^{-13}$ & 1.4 & $6.4 \times 10^{-14}$ \\
$\mathrm{~B}$ & $2^{d} \times(25 \times 360)$ & $99 \pm 6$ & $6 \pm 1$ & $<1.1$ & $-3.3 \pm 0.2$ & $<-1.4$ & $1.6 \times 10^{-14}$ & 0.4 & $5.6 \times 10^{-15}$ \\
\hline
\end{tabular}

${ }^{a}$ Minimum energy density.

${ }^{b}$ Equipartition magnetic field.

${ }^{c}$ Equipartition pressure.

${ }^{d}$ The tail is shaped as a double cylinder.

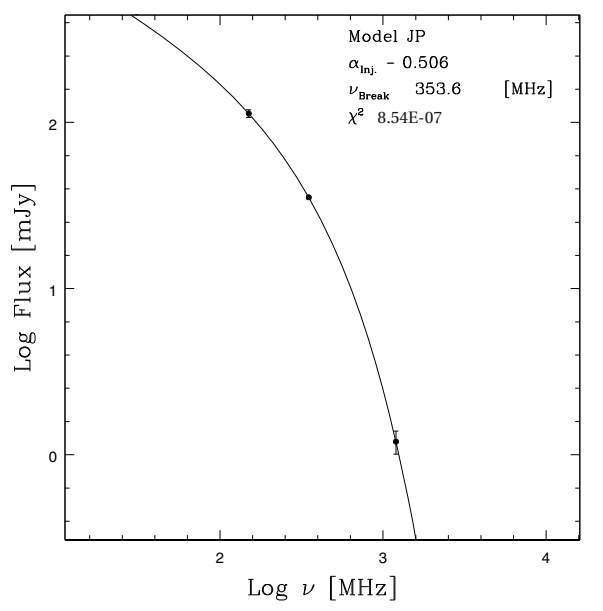

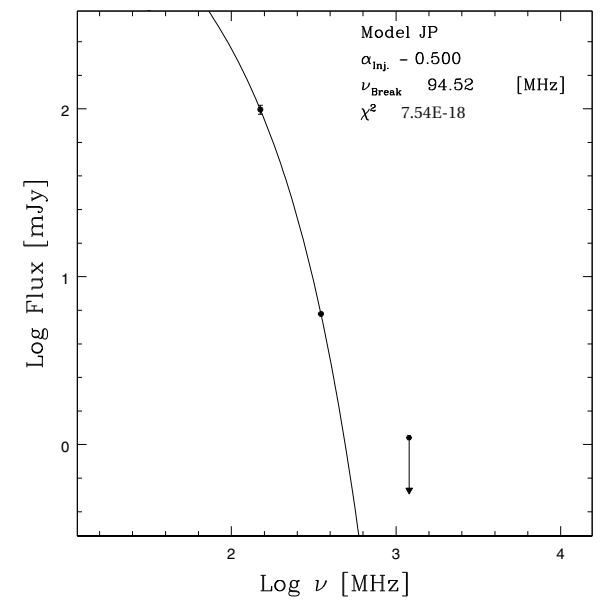

Fig. 18. Fluxes of region A (left panel) and region B (right panel) fitted with the JP model. $\chi^{2}$ represents the reduced $\chi^{2}$.
The X-ray emitting gas plays an important role in influencing the expansion and the structure of a radio source because it gives rise to a static pressure $\left(P_{\mathrm{st}}\right)$, which depends only on the gas temperature $\left(T_{\mathrm{ig}}\right)$ and on the numerical density $\left(n_{\mathrm{ig}}\right)$ of the X-ray emitting gas

$P_{\text {st }}=2 n_{\text {ig }} k T_{\text {ig }}$,

where $k$ is the Boltzmann constant.

For our analysis, we used the X-ray results obtained by Feretti et al. (1997), and we scaled the parameters to our cosmology. The central electron density of the X-ray emitting gas is $n_{0}=2.05 \times 10^{-3} \mathrm{~cm}^{-3}$ and its temperature is $\sim 3.5 \mathrm{keV}$, corresponding to $3.5 \times 10^{7} \mathrm{~K}$. The thermal pressure of the X-ray emitting gas has been scaled with the $\beta$ model law:

$n(r)=\frac{n_{0}}{\left[1+\left(\frac{r}{r_{\mathrm{c}}}\right)^{2}\right]^{3 \beta / 2}}$,

where $r_{\mathrm{c}}=4.8^{\prime} \pm 0.4^{\prime}$ and $\beta=0.74 \pm 0.04$.

The Beaver radio galaxy lies at a projected distance from the center of the cluster of $\sim 18^{\prime}(1.6 \mathrm{Mpc})$, where the X-ray brightness of the thermal gas is very low, but still above the background (see Table 8). By comparing the internal pressure of regions $\mathrm{A}$ and $\mathrm{B}$ along the tail (see Table 7) with the static pressure of the X-ray emitting gas at their projected distance from the cluster center computed with Eq. (14) (Table 8), we conclude that the equipartition pressure is lower by a factor of $10^{2}$ or $10^{3}$ than the corresponding thermal pressure of the gas. We notice that projection effects might play an important role in the determination of $P_{\text {st }}$. The large gap between $P_{\text {eq }}$ and $P_{\text {st }}$ implies that either the numerous assumptions used in the calculations of the equipartition parameters are not valid, or that there is a real deviation from the equipartition conditions.

\section{Discussion}

\subsection{Halo origin}

The origin and evolution of cluster radio halos is still a matter of debate (Jaffe 1977; Dennison 1980). The main difficulty in explaining their nature arises from their large size $(\sim 1 \mathrm{Mpc})$ and the short radiative lifetime of the relativistic electrons emitting in them. Different theoretical models have been suggested in order to infer the mechanism of transferring energy into the relativistic electron population and for the origin of the relativistic electrons themselves: in-situ re-acceleration of relativistic electrons by shock waves (primary models), particle injection from radio galaxies, acceleration out of the thermal pool, secondary electrons resulting from hadronic collisions of relativistic protons with the ICM gas protons (secondary models), and combination of these processes (Brunetti 2003; Blasi 2003; Petrosian 2003).

Several properties make the halo of A2255 unique among the other known cluster radio halos. Embedded in the halo emission there is an unusually large number (4) of tailed radio sources, which are likely supplying it with relativistic particles (Feretti et al. 1997). Morphologically, the structure of the halo is rather complex, as Figs. 4-7 show. At $25 \mathrm{~cm}$, the halo has a rectangular shape, due to the presence of 3 bright filaments at the edges, perpendicular to each other. Previous VLA observations at $1.4 \mathrm{GHz}$ (Govoni et al. 2005) revealed that they are strongly polarized $(\sim 20 \%-40 \%)$, which represents the first detection of a polarized halo in the literature. Also at $85 \mathrm{~cm}$ and at $2 \mathrm{~m}$ the halo has a rectangular shape, with a considerable extension towards the S and SW with respect to high-frequency (see Figs. 6 and 7). Furthermore, in the $2 \mathrm{~m}$ observations a new extended emitting region is detected to the NW of the halo (Figs. 7 and 8). This new feature, undetected in the more sensitive observations at $85 \mathrm{~cm}$ (Fig. 6), should have a very steep spectrum $(\alpha \leq-2.6)$. 
Table 8. Physical parameters of the X-ray emitting gas at the location of the Beaver.

\begin{tabular}{cccc}
\hline \hline Region & $\begin{array}{c}r^{a} \\
(\mathrm{kpc})\end{array}$ & $\begin{array}{c}n(r)^{b} \\
\mathrm{~cm}^{-3}\end{array}$ & $\begin{array}{c}P_{\mathrm{st}^{c}} \\
\left(\text { dyne cm }^{-2}\right)\end{array}$ \\
\hline Head & $1.5 \times 10^{3}$ & $1 \times 10^{-4}$ & $1.2 \times 10^{-12}$ \\
A & $1.1 \times 10^{3}$ & $2.2 \times 10^{-4}$ & $2.5 \times 10^{-12}$ \\
B & $7.8 \times 10^{2}$ & $4.1 \times 10^{-4}$ & $4.6 \times 10^{-12}$ \\
\hline
\end{tabular}

${ }^{a}$ Projected distance from the $\mathrm{X}$-ray centroid.

${ }^{b}$ Electron density.

${ }^{c}$ Static pressure.

As a consequence of the presence of the filaments, the surface brightness of the halo increases from the center towards the edges and this reflects in the the spectral index behavior within the halo (see Figs. 9-10). Although the low resolution of the images does not allow us to obtain very precise trends and the presence of the radio galaxies at the cluster center partially contaminate the determination of the spectral index, it is evident that $\alpha$ is steeper in the regions in between the filaments and flattens along them, where fresh (re-) acceleration of the relativistic particles takes place. This trend, due to the presence of the radio filaments, has not been seen before in a radio halo; in the few clusters for which maps of the spectral index are available (e.g. Coma C, Giovannini et al. (1993), A665, A2163, Feretti et al. 2004, A3562, Giacintucci et al. 2005, A2744, and A2219, Orrú et al. 2007) the halo radio spectrum shows variations on small scales (as clumpy structures) and/or on large scales (radial steepening from the center to the edges).

The singular properties of the halo of A2255 (morphology, surface brightness, spectral index distribution, and polarization), suggest that either this extended structure has a more complex structure than the other known radio halos or that it is seen in a particular stage of its evolution. Since our observations do not support a unique explanation for the origin of the radio halo, we suggest the two following scenarios:

- the halo and the filaments are two physically distinct structures, seen in projection as a unique feature.

$\mathrm{X}$-ray, optical and radio data provide evidence that A2255 is currently in an active dynamical state, and other surrounding structures might be interacting with it. A ROSAT X-ray survey observation indicates that this cluster belongs to the north ecliptic pole super-cluster, which contains at least 21 members (Mullis et al. 2001). Signs of the interaction of A2255 with the other cosmic structures are expected. Indeed, many radio features are detected at various distances from the cluster center. These radio "relics" suggest that the cluster is currently accreting gas from the cosmic environment and is possibly hosting many shock waves deriving from the past merger activity. Relics, usually found at the cluster periphery, should occasionally also be detected in projection towards the cluster center. Thus, it is possible that the three filaments that "form" the radio halo of A2255 are shocked regions of plasma which are seen in projection on the central real radio halo. Their highly polarized flux (Govoni et al. 2005) favors a location of these structures in the foreground instead than in the background, where Faraday depolarization would rapidly depolarize them. The new extended feature detected at $2 \mathrm{~m}$ to the NW of the cluster center could be considered as an extension of the halo and its very steep spectral index could be explained in the framework of the primary electron re-acceleration models, where a steepening of the spectral index from the center to the edges of the halo is expected. However, since radio halos in the literature are known to have regular morphologies, we should also consider the hypothesis that the new feature is not physically related with the central radio halo. In this case, this could be considered as the first example of Mpc-size diffuse structures (MDS), which lie around clusters and are detectable at very low frequency only.

\section{- the halo has an intrinsic surface brightness increasing towards the edges and a spectral index flattening from the center to its outermost regions.}

The bright and polarized filaments at the edge of the halo could derive from the injection in the intra cluster medium of energy on large scales, which is produced by turbulence, resulting from past merger activity. On the theoretical point of view, the picture of the development of turbulence in clusters seems still uncertain. Dolag et al. (2005) argue that the bulk of turbulence is injected in the core of galaxy clusters, implying a more developed turbulence in the innermost regions, compared to the outer parts. On the other hand, cosmological numerical simulations suggest that turbulence is expected to be greater at increasing radial distances from the cluster center (Bryan \& Norman 1998; Sunyaev et al. 2003). Moreover, Govoni et al. (2006) showed that to reproduce the $\mathrm{RM}$ distribution for three of the radio galaxies of A2255 one needs a power spectrum of the magnetic field of the cluster steepening from the center towards the periphery and the presence of filamentary structures on large scale. Therefore, it is possible that the turbulence at the center of A2255 gave rise to shocked regions at the edges of the halo.

In this framework, a physical connection between central halo and the newly detected extended feature to the NW from it seems unlikely. Thus, the latter should be classified as the first example of MDS.

\subsection{Relics}

Relics are associated with clusters with or without cooling core, suggesting that they may be related to minor or off-axis mergers, as well as major mergers. Their formation is supposed to be related to shocks either by Fermi-I diffuse acceleration of ICM electrons (Enßlin et al. 1998; Keshet et al. 2004a) or by adiabatic energization of the relativistic electrons confined in bubbles of fossil radio plasma ("ghosts"), released by a former active radio galaxy. Shocks in clusters environments can derive from the merger of subclusters ( merger shocks, Bykov et al. 2008), or can be due to the accretion of diffuse, unprocessed (cold) matter on gravitationally attracting nodes (accretion shocks, Miniati 2003; Keshet et al. 2003)

The presence of shocked regions in A2255 is strongly supported by X-ray and radio observations. Recently, the XMM-Newton satellite detected a shocked region near $\left(\sim 4^{\prime}\right.$, $360 \mathrm{kpc}$ ) the cluster center. The morphology of this thermal emission suggests that it could arise from a merger that happened along the east-west direction about $0.15 \mathrm{Gyr}$ ago (Sakelliou \& Ponman 2006). However, the complicated structure of the temperature map does not allow any precise conclusion about the geometry of the merger.

At radio wavelengths, A2255 shows the presence of several relics and filamentary features (see Figs. 4 and 6) which are likely associated with shocks. The young merger event 
inferred by the X-ray observations cannot be responsible for the formation of these features, which are located at large distance from the cluster center. The shock derived from it lies still inside the radio halo. Cosmological simulations show that cosmic structures form through several merger events. Consequently, the cluster environment can host many shocks traveling towards different directions, together with possible other shocks, appearing at large distance from the cluster center and possibly associated with the flow of cold gas into the potential wall of the cluster itself. This scenario seems to be present in A2255. In its outermost regions there are relic-like structures, which are probably associated with LSS shocks (Pizzo et al. 2008). In addition, in its innermost regions there are shock-related features (NE relic, bridge, $C 1$, and $C 2$ in Fig. 4) which likely derive from the past merger activity.

The shape and location of the NE relic clearly suggest that this could originate in a shock wave traveling along the NE to SW direction. The orientation of the magnetic field in it, being parallel to the major axis (Govoni et al. 2005), also supports this scenario. Its radio spectral index $(\alpha \sim-0.7)$, unusually flat for a relic, suggests that this structure is young and the relativistic electrons have been recently (re)accelerated. The spectral index trend along the minor axis, being steep near the cluster center and flattening towards the periphery (see left panel of Fig. 11), implies that the shock is traveling outwards. The fact that we see a gradient of the spectral index also along the major axis (see right panel of Fig. 11), may be due to the complex geometry of the shock. The presence of filamentary radio emission perpendicular to the NE relic (bridge, $C 1$ and $C 2$ in Fig. 4) suggests that another shock could be present at this location and it could have influenced the physical properties of the NE relic itself.

The nature of the NW and SW relics has been already investigated by Pizzo et al. (2008) by means of the $85 \mathrm{~cm}$ observations. The spectral index study carried out in this paper, allow us to reinforce our first conclusion. The spectrum of the NW relic has been determined for the only filament oriented towards the cluster center. Its $\alpha$ values $(-1.2 \pm 0.08 \leq \alpha \leq-0.9 \pm 0.1)$ and its location with respect to the cluster center, still support the hypothesis that it could be related to LSS shocks. The SW relic is not detected at $2 \mathrm{~m}$, therefore we can only compute a lower limit $(\alpha \geq-1.2)$. Given the common physical properties with the NW relic, we still suggest that also the SW relic could be associated with LSS shocks.

\subsection{The Beaver radio galaxy}

Narrow angle tail (NAT) radio galaxies have a U shape morphology, with the nucleus coincident with the parent optical galaxy and both tails bending backwards. Most of these sources were detected in clusters of galaxies and they have been the target of numerous investigations. The morphology of NAT radio sources is due to the ram pressure exerted by the ambient medium on the radio plasma ejected by the host galaxy that moves through the cluster. In this scenario, the quasi continuous beams ejected by the central galaxy are bent backwards, forming a tail which marks the path that the galaxy has traveled through the ICM. It is currently accepted that in non collapsing clusters, the motion of the ICM is subsonic, thus not having a substantial effect on the orientation of the radio tails. In this context, the tail of the radio galaxy will be parallel to the galaxy advance motion.

The size of NAT radio galaxies, from the nucleus till the end of the tail, is on average about 200-300 kpc (Vallee \& Roger 1987). This is comparable with the extent of the more common double radio sources, which have a I shape, with the parent galaxy located at the centroid. However, a few examples of NAT radio galaxies with uncommonly long tails are reported in the literature. Among them, the most well known objects are IC 711 in Abell 1314 ( 650 kpc, Vallee \& Roger (1987); Vallee (1988)) and NGC 1265 in the Perseus cluster (>500 kpc, Sijbring \& de Bruyn (1998)). The Beaver radio galaxy (1712+638, J2000), whose tail extends to almost $1 \mathrm{Mpc}$ at $85 \mathrm{~cm}$ wavelength, provides a clear example of such radio sources.

The long tail of the Beaver may suggest that the host galaxy (2MASX J17131603+6347378) has traveled from the central regions of the cluster to its actual location without any substantial deviation from its original direction, at least on the plane of the sky. Projection effects could make the tail appear shorter, but knowing the real extent of the tail as well as the 3-dimensional location of the Beaver with respect to the other cluster structures is challenging. A polarization study of the cluster at $85 \mathrm{~cm}$ reveals that the end of the tail of the Beaver is polarized (Pizzo et al., in prep.). This suggests that this structure is not located deep inside the dense X-ray emitting gas, which would make it depolarized due to Faraday depolarization. However, since the radial velocity of the galaxy $\left(v_{\text {Beaver }}=24843 \mathrm{~km} \mathrm{~s}^{-1}\right)$ is only $700 \mathrm{~km} \mathrm{~s}^{-1}$ higher than the mean velocity of the cluster $\left(v_{\mathrm{A} 2255}=24163 \mathrm{~km} \mathrm{~s}^{-1}\right)$, which is less than its velocity dispersion $\left(\sigma_{\mathrm{v}_{\mathrm{A} 2255}}=1266 \mathrm{~km} \mathrm{~s}^{-1}\right)$, the direction of the velocity vector cannot be determined.

We have checked that the long nature of the Beaver is real subtracting the point sources visible at higher frequency along the path of the tail in the low-frequency maps. Support to the genuine nature of the tail is also the regular steepening of the spectral index from the head towards its outermost regions (Figs. 14 and 15).

The severe steepening of the spectral index along the tail of the Beaver implies that the relativistic electrons responsible of the radio emission suffered important energy losses after their first ejection from the parent galaxy, which is confirmed by the high radiative ages of the plasma at the end of the tail $\left(\sim 2.2 \times 10^{8} \mathrm{yr}\right.$, see Sect. 5.2). Its detectability, even at low frequency, raises questions, since adiabatic expansion should play an important role in rapidly depriving the electrons of their energy. In this context, it is suggested that the ICM could have a drastic influence on the final stages of the life of the plasma (e.g. Venturi et al. 1998; Parma et al. 2007). In our case, the high static pressure exerted by the ICM on the ending part of the tail of the Beaver (where the internal pressure is $10^{3}$ times lower than the external one) may prevent its quick dissipation through adiabatic expansion.

It is worth noting that the Beaver shows at the end of the tail spectral index values very similar to the ones of the southern regions of the halo (Fig. 15). This characteristic suggests that the Beaver and the halo may be physically related structures and that the radio galaxy provides the halo with the relativistic particles for its own radio emission. Tailed radio sources are thought to supply the relativistic electrons to radio halos (see the example of NGC 4869 in the COMA cluster, Giovannini et al. 1993). In the Beaver radio galaxy and A2255 we may witness this process.

\section{Summary and conclusions}

We presented WSRT observations of the cluster of galaxies A2255 at wavelengths of $25 \mathrm{~cm}, 85 \mathrm{~cm}$, and $2 \mathrm{~m}$. In the final maps, the radio halo and the relics are detected. In each image, the radio emission associated with the cluster seems to be very complex and several new features are detected. Our observations, together with the results coming from optical and X-ray 
studies, have highlighted an interesting picture for this cluster, which has probably undergone multiple merger events during its past history. Several features, detected in the radio domain in and around the cluster, could be considered as an indication of a still undergoing strong dynamical activity.

At $25 \mathrm{~cm}$, the radio halo shows a $U$ shape, with three bright filaments perpendicular to each other at the edges. Two additional filamentary features are detected at low level, the first one at the same location of the NW relic found at $85 \mathrm{~cm}$ and the other one near the already known NE relic (see Fig. 4). At $85 \mathrm{~cm}$, the halo is more extended towards S and SW and at $2 \mathrm{~m}$ it shows a Mpc-size extension towards NW which was not detected previously.

From the spectral index images we found that $\alpha$ is steep in the central part of the halo and flattens moving towards its outermost regions (Figs. 9 and 10). This is likely due to the presence at this location of bright filaments, clearly detected at higher frequency. Understanding the nature of these filaments and of the newly detected extended emission between halo and NW relic is challenging. One possibility is that the central radio halo and the filaments are two physically unrelated structures, seen in projection near the cluster center. In this case, the extended feature detected at $2 \mathrm{~m}$ to the $\mathrm{NW}$ of the halo could be considered as an asymmetric extension of the halo itself; its steep spectrum ( $\alpha \leq-2.6$ ) could be justified in the framework of the primary electron re-acceleration models, where a steepening of the spectral index from the center to the edges of the halo is expected. However, since radio halos are known in the literature as structures showing a regular morphology, the new feature could be physically not related with the central radio halo and it should be considered as the first example of Mpc-size diffuse structures (MDS), which are detectable around clusters at very low frequency only. On the other hand, it is also possible that A2255 hosts an intrinsically peculiar radio halo, which has a filamentary structure at the edges. In this scenario, the diffuse emission region to the NW of the halo should be considered as not related to the central halo and classified as MDS. In order to distinguish between the two possible scenarios, it is important to understand the real nature of the filaments. Sensitive X-ray observations are needed to investigate the presence of X-ray substructures, possible shocks, and their connection to the radio halo. On the other hand, a detailed study of the rotation measure of the different physical structures of the cluster could make it possible to infer the 3-dimensional geometry of A2255 (Pizzo et al., in prep.), possibly answering this still open question.

The NE relic shows a flattening of the spectral index along its minor axis, moving outwards from the cluster center (see left panel of Fig. 11). This, and the rather flat integrated spectrum of the NE relic (Fig. 12, right panel), suggests that the relativistic particles have been recently (re) accelerated by a shock, that is traveling from the center of the cluster towards its periphery along the NE-SW direction.

The NW and SW relics are both detected at $85 \mathrm{~cm}$ only. The $\mathrm{NW}$ relic is also visible at $2 \mathrm{~m}$, but just partially at $25 \mathrm{~cm}$. From the spectral index image between $2 \mathrm{~m}$ and $85 \mathrm{~cm}$, we obtain spectral index values ranging between -0.6 and -1.8 , steepening towards the cluster center. We cannot obtain a direct estimation of the spectral index for the SW relic, because of sensitivity limitations in the $25 \mathrm{~cm}$ and $2 \mathrm{~m}$ maps, therefore only a lower limit can be derived in this case $(\alpha \geq-1.2)$.

The tail of the Beaver radio galaxy increases its length to almost $1 \mathrm{Mpc}$ between $25 \mathrm{~cm}$ and $85 \mathrm{~cm}$. This is a clear indication that the plasma along the tail suffered severe energy losses after the ejection from the parent optical galaxy, and that is still confined in the tail due to the static pressure exerted by the external ICM. The very long tail of the radio galaxy gives us the possibility to test ageing models for the relativistic electrons. The JP model gives a good representation of our data for the initial part of the tail (Fig. 18, left panel). For the ending part, more observations at intermediate wavelengths between $25 \mathrm{~cm}$ and $85 \mathrm{~cm}$ are needed to draw any conclusion (Fig. 18, right panel). In the spectral index map between $85 \mathrm{~cm}$ and $2 \mathrm{~m}$ (Fig. 15) there is the indication that the $\alpha$ values of the ending part of the tail of the Beaver are similar to the ones of the southern regions of the halo of A2255. This might suggest that the radio galaxy has provided the halo with relativistic particles for its own radio emission. To test this hypothesis, a more detailed study of the steepening of the spectral index along the tail of the Beaver is needed. LOFAR, thanks to its wide low-frequency range and high resolution, will play a major role in such investigations.

Acknowledgements. R.F.P. is thankful to Luigina Feretti for the useful discussions during the data analysis. R.F.P. is grateful to Monica Orienti for the very helpful and detailed comments and suggestions during the writing of the manuscript. The Westerbork Synthesis Radio Telescope is operated by ASTRON (Netherlands Institute for Radio Astronomy) with support from the Netherlands Foundation for Scientific Research (NWO).

\section{References}

Blasi, P. 2003, in ASP Conf. Ser. 301, ed. S. Bowyer, \& C.-Y. Hwang, 203 Blasi, P., \& Colafrancesco, S. 1999, Astroparticle Phys., 12, 169 Bonafede, A., Giovannini, G., Feretti, L., Govoni, F., \& Murgia, M. 2009, A\&A, 494, 429

Brunetti, G. 2003, in ASP Conf. Ser. 301, ed. S. Bowyer, \& C.-Y. Hwang, 349 Brunetti, G., Setti, G., \& Comastri, A. 1997, A\&A, 325, 898

Brunetti, G., Setti, G., Feretti, L., \& Giovannini, G. 2001, MNRAS, 320, 365 Brunetti, G., Blasi, P., Cassano, R., \& Gabici, S. 2004, MNRAS, 350, 1174 Bryan, G. L., \& Norman, M. L. 1998, ApJ, 495, 80

Burns, J. O., Roettiger, K., Pinkney, J., et al. 1995, ApJ, 446, 583

Bykov, A. M., Dolag, K., \& Durret, F. 2008, Space Sci. Rev., 134, 119

Cassano, R., \& Brunetti, G. 2005, MNRAS, 357, 1313

Cotton, W. D., Condon, J. J., Perley, R. A., et al. 2004, in SPIE Conf. Ser. 5489, ed. J. M. Oschmann, Jr., 180

Davis, D. S., Miller, N. A., \& Mushotzky, R. F. 2003, ApJ, 597, 202

Dennison, B. 1980, ApJ, 239, L93

Dolag, K., \& Enßlin, T. A. 2000, A\&A, 362, 151

Dolag, K., Vazza, F., Brunetti, G., \& Tormen, G. 2005, MNRAS, 364, 753

Enßlin, T. A., \& Gopal-Krishna. 2001, A\&A, 366, 26

Enßlin, T. A., Biermann, P. L., Klein, U., \& Kohle, S. 1998, A\&A, 332, 395

Evrard, A. E., \& Gioia, I. M. 2002, in Merging Processes in Galaxy Clusters, ed. L. Feretti, I. M. Gioia, \& G. Giovannini, Ap\&SS Library, 272, 253

Feretti, L., \& Giovannini, G. 1996, in Extragalactic Radio Sources, ed. R. D. Ekers, C. Fanti, \& L. Padrielli, IAU Symp., 175, 333

Feretti, L., Boehringer, H., Giovannini, G., \& Neumann, D. 1997, A\&A, 317, 432

Feretti, L., Orrù, E., Brunetti, G., et al. 2004, A\&A, 423, 111

Fujita, Y., Takizawa, M., \& Sarazin, C. L. 2003, ApJ, 584, 190

Garrett, M. A., de Bruyn, A. G., Giroletti, M., Baan, W. A., \& Schilizzi, R. T. 2000, A\&A, 361, L41

Giovannini, G., Feretti, L., \& Stanghellini, C. 1991, A\&A, 252, 528

Giovannini, G., Feretti, L., Venturi, T., Kim, K.-T., \& Kronberg, P. P. 1993, ApJ, 406, 399

Giacintucci, S., Venturi, T., Brunetti, G., et al. 2005, A\&A, 440, 867

Govoni, F., \& Feretti, L. 2004, Int. J. Mod. Phys. D, 13, 1549

Govoni, F., Murgia, M., Feretti, L., et al. 2005, A\&A, 430, L5

Govoni, F., Murgia, M., Feretti, L., et al. 2006, A\&A, 460, 425

Harris, D. E., Kapahi, V. K., \& Ekers, R. D. 1980, A\&AS, 39, 215

Harris, F. J. 1978, in Institute of Electrical and Electronics Engineers, Inc. Conf.,

Proc. IEEE, 66, 51

Högbom, J. A. 1974, A\&AS, 15, 417

Horner, D. 2001, Ph.D. Thesis, University of Meryland

Intema, H. 2007, in From Planets to Dark Energy: the Modern Radio Universe. October 1-5 2007, The University of Manchester, UK. Published online at

SISSA, Proc. Sci., 101

Jaffe, W. J. 1977, ApJ, 212, 1 
Jaffe, W. J., \& Perola, G. C. 1973, A\&A, 26, 423

Kardashev, N. S. 1962, SvA, 6, 317

Keshet, U., Waxman, E., Loeb, A., Springel, V., \& Hernquist, L. 2003, ApJ, 585, 128

Keshet, U., Waxman, E., \& Loeb, A. 2004a, ApJ, 617, 281

Keshet, U., Waxman, E., \& Loeb, A. 2004b, New Astron. Rev., 48, 1119

Miller, N. A., \& Owen, F. N. 2003, AJ, 125, 2427

Miniati, F. 2003, MNRAS, 342, 1009

Mullis, C. R., Henry, J. P., Gioia, I. M., et al. 2001, ApJ, 553, L115

Navarro, J. F., Frenk, C. S., \& White, S. D. M. 1995, MNRAS, 275, 56

Orrú, E., Murgia, M., Feretti, L., et al. 2007, A\&A, 467, 943

Pacholczyk, A. G. 1970, Radio astrophysics. Nonthermal processes in galactic and extragalactic sources, Series of Books in Astronomy and Astrophysics, (San Francisco: Freeman)

Parma, P., Murgia, M., de Ruiter, H. R., et al. 2007, A\&A, 470, 875

Petrosian, V. 2001, ApJ, 557, 560

Petrosian, V. 2003, in ASP Conf. Ser. 301, ed. S. Bowyer, \& C.-Y. Hwang, 337
Pizzo, R. F., \& de Bruyn, A. G. 2008, in Extragalactic Jets: Theory and Observation from Radio to Gamma Ray, ed. T. A. Rector, \& D. S. De Young, ASP Conf. Ser., 386, 368

Pizzo, R. F., de Bruyn, A. G., Feretti, L., \& Govoni, F. 2008, A\&A, 481, L91

Rottgering, H. J. A., Wieringa, M. H., Hunstead, R. W., \& Ekers, R. D. 1997, MNRAS, 290, 577

Sakelliou, I., \& Ponman, T. J. 2006, MNRAS, 367, 1409

Sijbring, D., \& de Bruyn, A. G. 1998, A\&A, 331, 901

Struble, M. F., \& Rood, H. J. 1999, ApJS, 125, 35

Subrahmanyan, R., Beasley, A. J., Goss, W. M., Golap, K., \& Hunstead, R. W. 2003, AJ, 125, 1095

Sunyaev, R. A., Norman, M. L., \& Bryan, G. L. 2003, Astron. Lett., 29, 783

Tribble, P. C. 1993, MNRAS, 263, 31

Vallee, J. P. 1988, Ap\&SS, 149, 225

Vallee, J. P., \& Roger, R. S. 1987, AJ, 94, 1

Venturi, T., Bardelli, S., Morganti, R., \& Hunstead, R. W. 1998, MNRAS, 298, 1113

Yuan, Q., Zhou, X., \& Jiang, Z. 2003, VizieR Online Data Catalog, 214, 90053 